\title{
Aiding buoyancy driven flow and heat transfer features of converging and diverging trapezoidal cylinders
}

\author{
MALIK PARVEEZ ${ }^{1}$, AMIT DHIMAN ${ }^{2, *}$ and G A HARMAIN ${ }^{3}$ \\ ${ }^{1}$ Department of Chemical Engineering, National Institute of Technology Srinagar, Hazratbal 190 006, India \\ ${ }^{2}$ Department of Chemical Engineering, Indian Institute of Technology Roorkee, Roorkee 247 667, India \\ ${ }^{3}$ Department of Mechanical Engineering, National Institute of Technology Srinagar, Hazratbal 190 006, India \\ e-mail: amitdfch@iitr.ac.in; dhimuamit@rediffmail.com
}

MS received 2 June 2017; revised 30 January 2018; accepted 9 February 2018; published online 27 June 2018

\begin{abstract}
Numerical computations have been carried out to explore the influence of mixed convection heat transfer from heated trapezoidal geometries of two different configurations namely converging and diverging cylinders in a vertical domain. The recirculation length of the diverging cylinder is found to be more than that of the converging cylinder for all values of Re (5 to 40) considered in this study and this length decreases after introducing buoyancy effect. Drag coefficients decrease with increase in Re for a fixed Ri. However, drag increases for the increasing values of Ri (values considered up to 1). The drag coefficient is found to be the smaller for diverging cylinder than that of converging one. Local Nusselt number shows significant increase as $\mathrm{Re}$ and $\mathrm{Ri}$ values increase, which results in enhanced heat transfer. Keeping Ri fixed and increasing the value of Re results in the augmentation of heat transfer and is around $15 \%$ at $\mathrm{Re}=5$ and $23 \%$ at $\mathrm{Re}=40$ for $\mathrm{Ri}=0$ for a square cylinder with respect to diverging cylinder. Under the influence of aiding buoyancy, the values of average Nusselt number $(\overline{\mathrm{Nu}})$ for the diverging cylinder are higher compared to that of converging cylinder. A correlation expressing functional relationship of $\overline{\mathrm{Nu}}$ with Re and Ri has also been generated.
\end{abstract}

Keywords. Diverging cylinder; converging cylinder; aiding buoyancy; drag coefficients; Nusselt numbers.

\section{Introduction}

Examining fluid flow around bluff objects has remained a subject of immense importance for several decades. The trapezoidal bluff body is a typical configuration of vortex shedding bodies mounted on vortex flow meters and is widely used in other applications, such as electronic cooling, heat exchange systems, designing of various offshore structures such as oil buoys and floating structures, probes and sensors, etc.. New issues pertaining to aiding and opposing buoyancies have recently been investigated from numerical and experimental points of view. Although the contribution of forced convection is significant for higher Reynolds number (Re), the impact of natural convection becomes prominent at low Re. The value of Richardson number $\mathrm{Ri}=0$ is the indicative of the forced convection. The larger the value of Ri $(>0)$, free convection is considerable; with values greater than unity is generally indication of mixed convection. In a continuous medium with obstacles, natural convection effects need to be considered in presence of temperature gradients. The shape of the body has a significant effect on the transport phenomena

*For correspondence involving momentum and thermal effects. Furthermore, the orientation of the obstacles changes the flow conditions and it may also affect the heat transfer. It has been reported in the literature that buoyancy effect makes flow field complex for the low range of Re and affects the transfer of heat. Heat transfer from the surface is enhanced by buoyancy effects if these assist the forced flow, whereas the reverse effect is observed if they resist the flow. It is important to mention that the mixed convection heat transfer from a heated trapezoidal cylinder in a vertical domain has received virtually no attention.

The present work addresses the combined effects of forced and free convection based heat transfer from heated converging and diverging trapezoidal cylinders in Newtonian fluid with the buoyancy imposed on the flow field (aiding buoyancy).

\section{Previous investigations}

The issues of fluid flow and transfer of heat around an object (which including circular, square, rectangular and trapezoidal geometries) have been studied for various cases such as aiding, opposing, and cross buoyancies and 
reported in detail in open literature. The laminar convective heat transfer for a circular cylinder involving parallel and contra flow patterns (for Re values 5, 20, 40 and 60) with a variable Grashof number (Gr) was investigated in [1]. Numerical results on steady convection under aiding buoyancy (varying the upstream distance, total height and width with respect to diameter of cylinder) for Re values 20 to 60 and Ri up to 4 were reported in [2]. It was concluded that lesser the blockage effect, results in the enhancement of forced convection, but reduced considerably the buoyancy-induced heat transfer rate for the case of vertical channel. The mixed convection under cross flow arrangement from a circular cylinder covering $\operatorname{Re} 1$ to 40 for $\mathrm{Gr}=5 \mathrm{Re}^{2}$ at $\mathrm{Pr}=0.7$ was examined in [3]. A mathematical model was developed and tested with the problem of forced convection in the same range of Re. A good agreement was found on comparing the results with previous theoretical and experimental data. The effect of $\mathrm{Ri}$ was studied for a square geometry in the vertical cross flow condition for $\mathrm{Re}=100$ and $\mathrm{Pr}=0.7$ [4]. For $\mathrm{Ri} \leq 0.125$ the flow was found to be unsteady periodic, while that for $\mathrm{Ri} \geq 0.15$ the flow was steady. The transition to steady occurred between $\mathrm{Ri}=0.125$ and 0.15 . For $\mathrm{Ri} \geq-0.1$ the flow started separating at the trailing edge of the cylinder, while the onset of leading edge flow separation occurred between $\mathrm{Ri}=-0.1$ and -0.15 . The recirculation length increased with increase in Ri for periodic flow, while it decreased monotonically with the increase in Ri for steady flow. Finite volume algorithm was used to simulate mixed convective flow with circular geometry [5]. Two flow patterns had been identified at the critical value of $\mathrm{Ri}=0.15$. Large size vortices and reduced shedding frequency did not enhance heat transfer significantly while average Nusselt number $\overline{N u}$ increased monotonically with increasing Ri.

Wake formation behind square and circular cylinders studied experimentally for low Re [6]. Flow field was examined (forced to mixed convection) over the range of conditions: flow velocity $=0.13,0.23,0.27 \mathrm{~m} / \mathrm{s}$ with $\mathrm{Re}=53$, 94, 118, respectively. Investigation by [7] reported that Strouhal number increased slowly with Ri. Flow and heat transfer characteristics were also explored experimentally. Results were reported for eccentricities ranging from the concentric case to near-contact case at $\operatorname{Re}=1500,2800$, and 5700. There was minor influence on the total transfer of heat for small eccentricity up to about 0.3 . An experimental study involving different shapes of bluff bodies (with water as a working fluid) was conducted by [8]. Based on signal amplitude and consistency of Strouhal number, the trapezoidal bluff body was found more suitable in terms of clear separation of flow and vortex formation.

Square cylinder under the influence of aiding buoyancy for $\mathrm{Re}=1-40$ and $\mathrm{Ri}=0-1$ was investigated by [9]. The drag coefficient $C_{D}$ decreased with increase in Re, while a reverse trend was observed with increasing Ri. Further, the average Nusselt number increased with $\mathrm{Re}$ and/or $\mathrm{Ri}$. The effect of buoyancy on a circular cylinder in Newtonian/ power-law fluids was presented by [10]. Flow and heat transfer characteristics were studied under the effects of $0 \leq \mathrm{Ri} \leq 2,1 \leq \operatorname{Re} \leq 40$ and $1 \leq \operatorname{Pr} \leq 100$. A reduction in the wake size with increasing $\mathrm{Ri}$ and/or $\mathrm{Pr}$ was reported in that investigation.

Formation of the wake behind a converging cylinder was presented by [11] for Re in the range of 25 to 1000 . It was found that flow started without separation and as time progressed, the wake developed behind the trapezoidal body. Reference [12] covered an experimental study for the performance of converging-diverging vortex flow meter. The trapezoidal case was recommended for convergingdiverging vortex flow meter on the basis of thorough comparison of the overall performance of the trapezoidal and square bluff bodies. Reference [13] discussed the influence of height ratio on the vortex shedding from a trapezoidal body. The minimum value of Strouhal number was found at $\mathrm{Re}=100$ and 150 for height ratios of 0.7 and 0.85 , respectively. The flow separation occurred near the front corner at $\mathrm{Re}=200$. The comparative study on cross buoyancy mixed convection around converging and diverging cylinders was carried out for $\mathrm{Re}=10-50$, $\mathrm{Ri}=0-1$ and $\operatorname{Pr}=0.7$ [14]. Overall drag and lift coefficients were found to be the higher for the converging cylinder as compared with the diverging cylinder. On increasing $\mathrm{Ri}$, overall coefficients (drag and lift) were found to decrease in the steady regime for both cylinders. The flow around a confined trapezoidal cylinder was inspected for $\operatorname{Pr}=0.71$ and $\mathrm{Ri}=0-1$ in [15]. The critical values of Re (Reynolds number at which transition occurs from steady to periodic) attained were 37,61 and 92 for the blockages of 12.5, 25 and 50\%, respectively. That study also reported relevant literature [16-18] encompassing the flow and heat transfer around trapezoidal cylinder.

The literature review presented here succinctly reveals that some problems $[4,6,9,14,15]$ related to (aiding and/ or cross buoyancy) mixed convection for square and other geometries are reported. However, the problem of aiding buoyancy mixed convection for the converging and diverging type of geometries is not explored. For instance study [4] presented the effect of $\mathrm{Ri}$ in cross flow configuration for a square cylinder, whereas the study [6] investigated the aiding buoyancy effect around the square and circular cylinders. A numerical study on aiding buoyancy for a square cylinder was detailed in [9]. In a recent study [14], cross buoyancy effects were reported for both converging and diverging cylinders. However, in another study [15], the blockage effects were carried out for a trapezoidal geometry in the confined framework. The heat transfer enhancement, correlations of wake length and Nusselt number as a function of $\mathrm{Ri}$ and $\mathrm{Re}$ and comparison of converging and diverging effects of the trapezoidal cylinder 


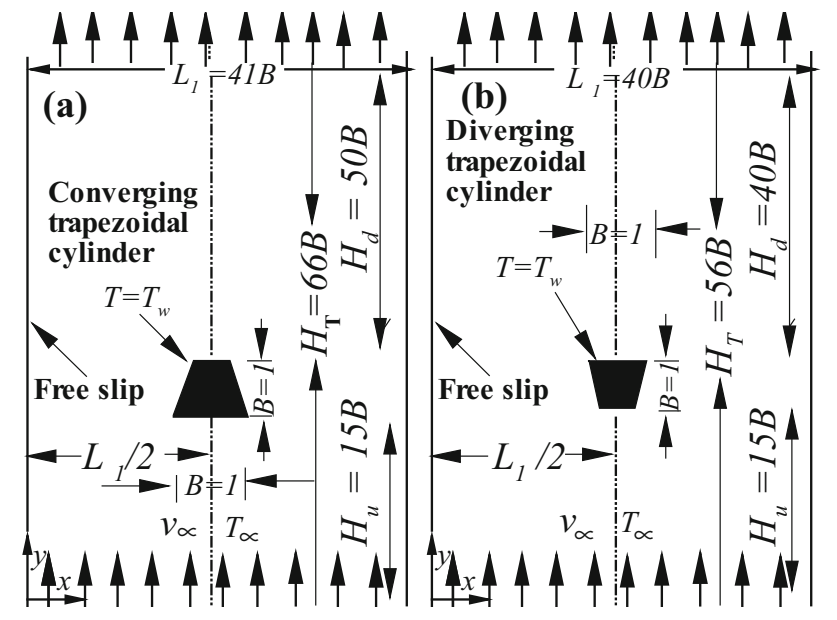

Figure 1. Schematics of (a) converging and (b) diverging trapezoidal cylinders.

under aiding buoyancy are new things which are explored in this study. Furthermore, the gaps in the literature especially around trapezoidal cylinders under the impact of aiding buoyancy in a vertical domain are the main focus of this study.

\section{Physical description of the problem}

The dimensions of the geometries considered for the analysis are given schematically in figure 1. Incompressible fluid flows over the long trapezoidal cylinders placed symmetrically in a vertical channel on the centre line. The domain is unconfined i.e., within two symmetric artificial boundaries. These trapezoidal cylinders are subjected to uniform free-stream in the upward direction represented as $v_{\infty}$ at a temperature $T_{\infty}$. Trapezoidal cylinders are at temperature $T_{w}\left(>T_{\infty}\right)$. The upstream distance is measured from the inlet of the domain to the front end of the trapezoidal cylinders i.e., ' $H_{u} / B$ ' (dimensionless) and downstream distance ' $H_{d} / B$ ' (dimensionless) from the rear end of the trapezoidal cylinders to the exit of the domain with total height of the computational domain as $H_{T} / B$. The total width of the domain is $L_{1} / B$ along $X$-axis.

The governing equations in dimensionless form are given below presuming insignificant viscous dissipation and constant thermo-physical properties.

Continuity equation:

$$
\frac{\partial U}{\partial X}+\frac{\partial V}{\partial Y}=0
$$

$X$-momentum equation:

$$
\frac{\partial U}{\partial \tau}+\frac{\partial(U U)}{\partial X}+\frac{\partial(V U)}{\partial Y}=-\frac{\partial P}{\partial X}+\frac{1}{\operatorname{Re}}\left(\frac{\partial^{2} U}{\partial X^{2}}+\frac{\partial^{2} U}{\partial Y^{2}}\right)
$$

$Y$-momentum equation:

$$
\frac{\partial V}{\partial \tau}+\frac{\partial(U V)}{\partial X}+\frac{\partial(V V)}{\partial Y}=-\frac{\partial P}{\partial Y}+\frac{1}{\operatorname{Re}}\left(\frac{\partial^{2} V}{\partial X^{2}}+\frac{\partial^{2} V}{\partial Y^{2}}\right)+R i \theta
$$

where $U=\frac{u}{v_{\infty}}, V=\frac{v}{v_{\infty}}, \tau=\frac{t v_{\infty}}{B}, X=\frac{x}{B}, Y=\frac{y}{B}, P=\frac{p}{\rho v_{\infty}^{2}}$,

$$
\operatorname{Re}=\frac{\rho v_{\infty} B}{\mu}, R i=\frac{G r}{\operatorname{Re}^{2}} \text { with } G r=\frac{g \beta\left(T_{w}-T_{\infty}\right) B^{3}}{v_{\infty}^{2}}
$$

The boundary conditions (dimensionless) are:

Inlet (bottom): $U=0, V=1$ Artificial boundary (right and left): $\frac{\partial V}{\partial X}=0, U=0$

Surface of the object: $U=0, V=0$ Exit (top): $\frac{\partial U}{\partial Y}=0, \frac{\partial V}{\partial Y}=0$

Energy equation:

$$
\frac{\partial \theta}{\partial \tau}+\frac{\partial U \theta}{\partial X}+\frac{\partial V \theta}{\partial Y}=\frac{1}{\operatorname{Re} \operatorname{Pr}}\left(\frac{\partial^{2} \theta}{\partial X^{2}}+\frac{\partial^{2} \theta}{\partial Y^{2}}\right)
$$

where $\theta=\left(\frac{T-T_{\infty}}{T_{w}-T_{\infty}}\right)$ and $\operatorname{Pr}=\mu \mathrm{C}_{p} / k$

The thermal boundary conditions (dimensionless) are:

Inlet (bottom): $\theta=0$ Exit (top): $\frac{\partial \theta}{\partial Y}=0$

Artificial boundary (right and left): $\frac{\partial \theta}{\partial X}=0$ Surface of the object: $\theta=1$

Equations (1) through (4) in conjunction with above boundary conditions are numerically solved using Ansys Fluent [19] as per the following methodology.

\section{Numerical methodology}

Commercial finite volume solver Ansys Fluent [19] has been used for solving the problem by invoking SIMPLE algorithm on the collocated grid arrangement. The computational grid has been generated with a coarse grid except in the vicinity of trapezoidal cylinders where fine meshing has been implemented as shown in figure 2. Discretization of convective terms has been performed using second-order upwind technique and diffusive terms discretised by using central difference method. Gauss-Siedel point-by-point iterative scheme has been used in conjunction with algebraic multi grid solver. The absolute residuals are fixed as $10^{-10}$ for continuity and velocity, whereas an absolute residual of $10^{-15}$ is fixed for energy.

\subsection{Grid independence test}

Grid sensitivity study was performed for both converging and diverging trapezoidal cylinders (table 1). The total number of cells used are 91640, 104010 and 119850 with control volumes (CVs) 100, 125 and 150 over the surface of diverging trapezoidal cylinder, respectively. Similarly, the total number of cells used for converging trapezoidal cylinder are 93950, 104720 and 122250 with CVs 100, 125 and 150 , respectively. This study was carried out for $\mathrm{Re}=40$ and $\mathrm{Ri}=0$, 1 , with dimensionless width $L_{1} /$ 
(a)

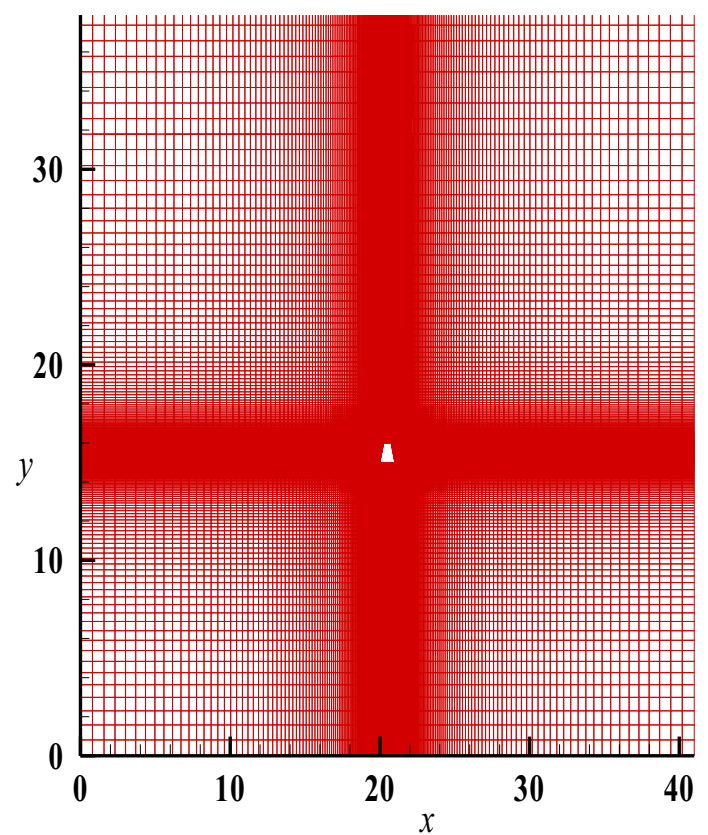

(b)

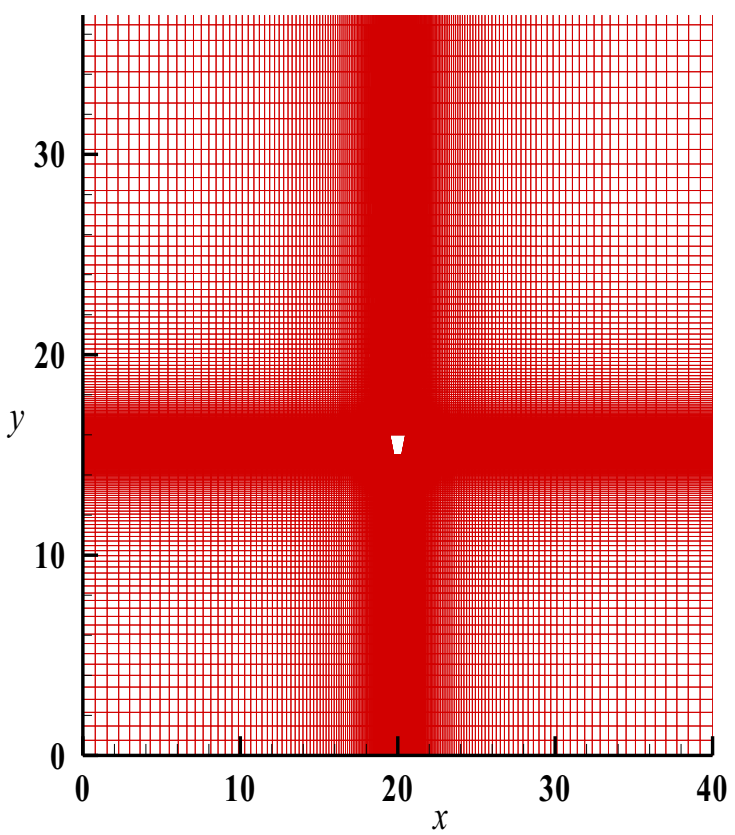

Figure 2. Non-uniform computational grid used for (a) converging and (b) diverging trapezoidal cylinders.

Table 1. Grid independence test for $\mathrm{Ri}=0,1$ at $\mathrm{Re}=40$ and $\mathrm{Pr}=0.71$ for diverging and converging trapezoidal cylinders

\begin{tabular}{|c|c|c|c|c|c|c|c|}
\hline $\mathrm{Ri}$ & Total no. of cells & $\mathrm{CV}$ on each side of trapezoidal cylinder & Smallest grid size, $\delta$ & $\mathrm{C}_{\mathrm{D}}$ & $\%$ variation & $\overline{N u}$ & $\%$ variation \\
\hline \multicolumn{8}{|c|}{ Diverging cylinder } \\
\hline \multirow[t]{3}{*}{0} & 91640 & 100 & 0.01 & 1.4895 & 0.03 & 3.3793 & 0.05 \\
\hline & 104010 & 125 & 0.008 & 1.4900 & - & 3.3776 & - \\
\hline & 119850 & 150 & 0.007 & 1.4903 & 0.02 & 3.3767 & 0.03 \\
\hline \multirow[t]{3}{*}{1} & 91640 & 100 & 0.01 & 3.4269 & 0.09 & 3.9341 & 0.06 \\
\hline & 104010 & 125 & 0.008 & 3.4301 & - & 3.9316 & - \\
\hline & 119850 & 150 & 0.007 & 3.4318 & 0.05 & 3.9304 & 0.03 \\
\hline \multicolumn{8}{|c|}{ Converging cylinder } \\
\hline \multirow[t]{3}{*}{0} & 93950 & 100 & 0.01 & 1.6542 & 0.08 & 2.5181 & 0.06 \\
\hline & 104720 & 125 & 0.008 & 1.6555 & - & 2.5166 & - \\
\hline & 122250 & 150 & 0.007 & 1.6561 & 0.04 & 2.5160 & 0.02 \\
\hline \multirow[t]{3}{*}{1} & 93950 & 100 & 0.01 & 3.0437 & 0.10 & 3.1845 & 0.05 \\
\hline & 104720 & 125 & 0.008 & 3.0468 & - & 3.1829 & - \\
\hline & 122250 & 150 & 0.007 & 3.0483 & 0.05 & 3.1822 & 0.02 \\
\hline
\end{tabular}

$B=41$ for height $H_{T} / B=66$ in case of converging cylinder. While $L_{1} / B=40$ and $H_{T} / B=56$ were used for the diverging cylinder. There is no prominent variation in the value of $\overline{N u}$ with a maximum deviation of about $0.06 \%$ for both geometries, with $\mathrm{Re}=40$ at $\mathrm{Ri}=0$. There is a maximum deviation of $0.06 \%$ in the value of $\overline{N u}$ for both geometries at $\mathrm{Ri}=1$ with $\mathrm{Re}=40 . \mathrm{C}_{\mathrm{D}}$ had the maximum deviation of about $0.08 \%$ for both the geometries with $\mathrm{Ri}=0$ at $\mathrm{Re}=40$. Further, the maximum deviation in the value of $C_{D}$ had been found to be around $0.1 \%$ for the converging and diverging cylinders for same conditions.
After a thorough analysis of deviations in the values of engineering parameters, the present study is carried out on the grid sizes of 104010 and 104720 for both geometries, with $125 \mathrm{CVs}$.

\subsection{Domain independence test}

The influence of varying upstream distance on the parameters such as drag coefficient $C_{D}$ and average Nusselt number $\overline{N u}$ is carried out for the two dimensionless values 
Table 2. Upstream domain independence test for $\mathrm{Ri}=0,1$ at $\mathrm{Re}=5$ and $\mathrm{Pr}=0.71$ for diverging and converging trapezoidal cylinders.

\begin{tabular}{lcccccc}
\hline Output parameter & $H_{u} / B=15$ & $H_{u} / B=20$ & $\%$ variation & $H_{u} / B=15$ & $H_{u} / B=20$ & $\%$ variation \\
\hline Diverging cylinder & & $\mathrm{Ri}=0$ & & & $\mathrm{Ri}=1$ & \\
$\mathrm{C}_{\mathrm{D}}$ & 4.2104 & 4.1680 & 1.02 & 9.3583 & 9.3824 & 0.26 \\
$\mathrm{Nu}$ & 1.3992 & 1.3953 & 0.28 & 1.6445 & 1.6462 & 0.10 \\
Converging cylinder & & & & & & \\
$\mathrm{C}_{\mathrm{D}}$ & 4.2588 & 4.2113 & 1.13 & 9.0221 & 9.0474 & 0.28 \\
$\mathrm{Nu}$ & 1.2212 & 1.2180 & 0.26 & 1.4470 & 1.4483 & 0.09 \\
\hline
\end{tabular}

Table 3. Downstream domain independence test for $\mathrm{Ri}=0,1$ at $\mathrm{Re}=40$ and $\mathrm{Pr}=0.71$ for diverging and converging trapezoidal cylinders.

\begin{tabular}{|c|c|c|c|c|c|c|}
\hline Output parameter & $H_{d} / B=41$ & $H_{d} / B=46$ & $\%$ variation & $H_{d} / B=41$ & $H_{d} / B=46$ & $\%$ variation \\
\hline \multicolumn{7}{|l|}{ Diverging cylinder } \\
\hline & & $\mathrm{Ri}=0$ & & & $\mathrm{Ri}=1$ & \\
\hline $\mathrm{C}_{\mathrm{D}}$ & 1.4900 & 1.4900 & 0 & 3.4300 & 3.4301 & 0 \\
\hline$\overline{N u}$ & 3.3776 & 3.3776 & 0 & 3.9316 & 3.9316 & 0 \\
\hline \multicolumn{7}{|c|}{ Converging cylinder } \\
\hline $\mathrm{C}_{\mathrm{D}}$ & 1.6555 & 1.6555 & 0 & 3.0468 & 3.0468 & 0 \\
\hline$\overline{N u}$ & 2.5166 & 2.5166 & 0 & 3.1829 & 3.1829 & 0 \\
\hline
\end{tabular}

Table 4. Domain width independence test for $\mathrm{Ri}=0,1$ at $\mathrm{Re}=40$ and $\operatorname{Pr}=0.71$ for diverging and converging trapezoidal cylinders.

\begin{tabular}{|c|c|c|c|c|c|c|}
\hline Output parameter & $L_{1} / B=40$ & $L_{1} / B=45$ & $\%$ variation & $L_{1} / B=40$ & $L_{l} / B=45$ & $\%$ variation \\
\hline \multicolumn{7}{|l|}{ Diverging cylinder } \\
\hline & & $\mathrm{Ri}=0$ & & & $\mathrm{Ri}=1$ & \\
\hline $\mathrm{C}_{\mathrm{D}}$ & 1.4900 & 1.4882 & 0.12 & 3.4301 & 3.4340 & 0.11 \\
\hline$\overline{N u}$ & 3.3776 & 3.3766 & 0.03 & 3.9316 & 3.9331 & 0.04 \\
\hline Output parameter & $L_{l} / B=41$ & $L_{l} / B=51$ & $\%$ variation & $L_{l} / B=41$ & $L_{l} / B=51$ & $\%$ variation \\
\hline \multicolumn{7}{|l|}{ Converging cylinder } \\
\hline $\mathrm{C}_{\mathrm{D}}$ & 1.6555 & 1.6521 & 0.20 & 3.0468 & 3.0532 & 0.21 \\
\hline$\overline{N u}$ & 2.5166 & 2.5158 & 0.03 & 3.1829 & 3.1842 & 0.04 \\
\hline
\end{tabular}

of upstream distances $H_{u} / B=15,20$ at $\mathrm{Ri}=0,1$ with $\mathrm{Re}=5$. Table 2 gives the variation in the values of $\mathrm{C}_{\mathrm{D}}$ and $\overline{N u}$ as $1.02 \%, 0.28 \%$ for $\mathrm{Ri}=0$ and $0.26 \%, 0.10 \%$ for $\mathrm{Ri}=1$ respectively for diverging trapezoidal cylinder. In case of converging trapezoidal cylinder, the difference in the values of $\mathrm{C}_{\mathrm{D}}$ and $\overline{N u}$ has been found to be $1.13 \%$, $0.26 \%$ for $\mathrm{Ri}=0$ and $0.28 \%, 0.09 \%$ for $\mathrm{Ri}=1$, respectively.

Similarly in table 3 , the effect of varying dimensionless distance on the downstream side $H_{d} / B$ on $\mathrm{C}_{\mathrm{D}}$ and $\overline{N u}$ is carried out at $H_{d} / B=41,46$ for diverging cylinder and $H_{d} /$ $B=51,61$ for converging cylinder at $\mathrm{Ri}=0,1$ for $\operatorname{Re}=40$. The variation in $\mathrm{C}_{\mathrm{D}}$ and $\overline{N u}$ is found to be the marginal for both $\mathrm{Ri}(=0,1)$.
The aforementioned analysis shows that the dimensionless upstream distance of 15 is suitable for both converging and diverging trapezoidal cylinders. The dimensionless downstream distances of 51 and 41 are found suitable for converging and diverging trapezoidal cylinders, respectively.

The effect of varying domain width in case of the diverging trapezoidal cylinder is reported for $L_{1} / B=40,45$ at $\mathrm{Ri}=0,1$ for $\mathrm{Re}=40$ in table 4 . The deviations in $\mathrm{C}_{\mathrm{D}}$ and $\overline{N u}$ for diverging cylinder are found to be $0.12 \%, 0.03 \%$ for $\mathrm{Ri}=0$ and $0.11 \%, 0.04 \%$ for $\mathrm{Ri}=1$, respectively. For converging trapezoidal cylinder, this test is conducted for dimensionless widths 41 and 51 (table 4). Corresponding deviations in $\mathrm{C}_{\mathrm{D}}$ and $\overline{N u}$ are found to be $0.2 \%, 0.03 \%$ for 
Table 5. (a) Validation of present square cylinder results with literature $[9,20,21]$ for $\mathrm{Ri}=0$ at different Re and (b) Validation of present results with literature $[17,18]$ for $\mathrm{Ri}=0$ at different Re.

(a)

\begin{tabular}{|c|c|c|c|c|c|}
\hline Source & $\mathrm{Re}$ & $\mathrm{C}_{\mathrm{Dp}}$ & $\mathrm{C}_{\mathrm{Dv}}$ & $\mathrm{C}_{\mathrm{D}}$ & $\overline{N u}$ \\
\hline Present & 10 & 2.1900 & 0.9854 & 3.1754 & 1.5446 \\
\hline Sharma et al [9] & & 2.3555 & 0.9343 & 3.2898 & 1.5573 \\
\hline Dhiman et al [20] and Anjaiah et al [21] & & - & 0.9437 & 3.2599 & 1.5624 \\
\hline Present & 40 & 1.4193 & 0.2861 & 1.7054 & 2.6664 \\
\hline Sharma et al [9] & & 1.5317 & 0.2493 & 1.7809 & 2.6012 \\
\hline Dhiman et al [20] and Anjaiah et al [21] & & 1.5130 & 0.2538 & 1.7668 & 2.6969 \\
\hline
\end{tabular}

(b)

\begin{tabular}{|c|c|c|c|c|c|c|}
\hline \multicolumn{4}{|c|}{$\mathrm{C}_{\mathrm{D}}$} & \multicolumn{3}{|c|}{$\overline{N u}$} \\
\hline \multicolumn{7}{|c|}{ Converging cylinder } \\
\hline $\operatorname{Re}$ & Present & [17] & $\%$ variation & Present & {$[17]$} & $\%$ variation \\
\hline 5 & 4.2588 & 4.3220 & 1.46 & 1.2212 & 1.2130 & 0.67 \\
\hline 40 & 1.6555 & 1.6719 & 0.98 & 2.5166 & 2.4910 & 0.10 \\
\hline \multicolumn{7}{|c|}{ Diverging cylinder } \\
\hline $\mathrm{Re}$ & Present & [18] & $\%$ variation & Present & {$[18]$} & $\%$ variation \\
\hline 5 & 1.6914 & 1.7145 & 1.35 & 1.3992 & 1.4012 & 0.14 \\
\hline 30 & 2.9713 & 2.9761 & 0.16 & 1.6914 & 1.7145 & 1.35 \\
\hline
\end{tabular}

$\mathrm{Ri}=0$ and $0.21 \%, 0.04 \%$ for $\mathrm{Ri}=1$, respectively. Finally, the dimensionless domain widths of 41 and 40 are found suitable for converging and diverging trapezoidal cylinders respectively.

\section{Results and discussion}

Computations have been carried out at $\operatorname{Re}=5-40$, $\mathrm{Ri}=0-1$ and $\operatorname{Pr}=0.71$ (air) in the unconfined vertical domain with free slip and aiding buoyancy conditions.

Table 5a gives the comparison between the present work and those available from literature [9, 20, 21] and an excellent correspondence exists between these results. Table 5a gives differences in $\mathrm{C}_{\mathrm{D}}$ as $3.5 \%, 2.6 \%$ and for $\overline{\mathrm{Nu}}$ the corresponding variations are $0.8 \%, 1.1 \%$ with respect to available values $[9,20,21]$ at $\mathrm{Ri}=0$ with $\mathrm{Re}=10$. Further, keeping all the conditions identical to those available in $[9,20]$, the relative differences in the values of $\mathrm{C}_{\mathrm{D}}$ are found to be $4.2 \%, 3.5 \%$ and for $\overline{N u}$ the variations are $2.5 \%$, $1.1 \%$ at $\mathrm{Ri}=0$ for $\mathrm{Re}=40$. The present work is also validated with analogous geometry available [17, 18] and the variations are found to be within reasonable limits, as provided in table $5 \mathrm{~b}$.

\subsection{Wake length and flow patterns}

Figure 3 presents the variation of dimensionless recirculation length $L r / B$ with $\operatorname{Re}$ in the steady flow for different Richardson number for both diverging and converging trapezoidal cylinders. At this juncture, recirculation length

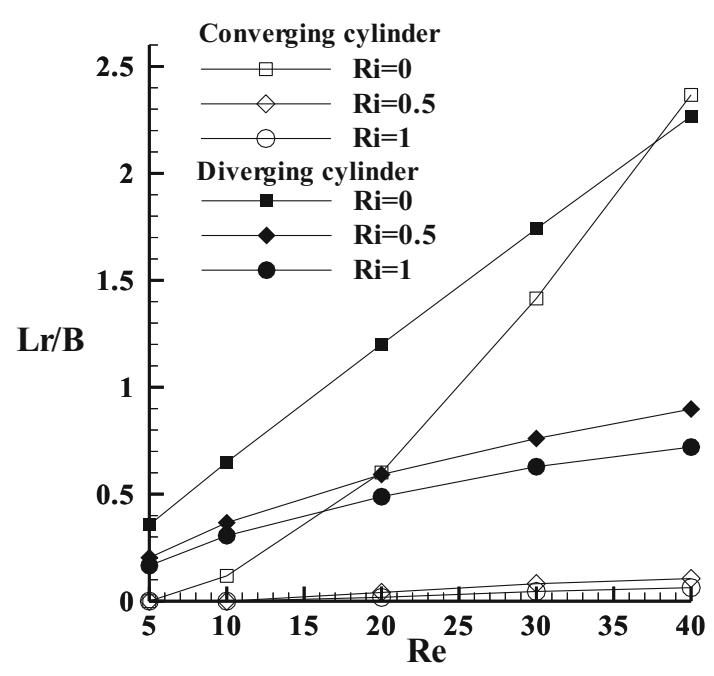

Figure 3. Variation of wake length with $\mathrm{Re}$ and $\mathrm{Ri}$ for converging and diverging trapezoidal cylinders.

(or wake length) is expressed as the distance measured from the rear of the trapezoidal cylinders to the reattachment point along the centreline. It is observed that recirculation length increases monotonically with varying Re from 5 to 40 for all values of $\mathrm{Ri}$. Recirculation length for the diverging trapezoidal cylinder is found to be comparatively higher than that of the converging trapezoidal cylinder at all values of Re and Ri used in this study. It has been observed from the study that after introducing the effect of Ri, wake length decreases significantly. A non-linearity in wake length is observed due to the incorporation of aiding 
(a) $\mathbf{R i}=\mathbf{0}, \mathbf{R e}=\mathbf{5}$

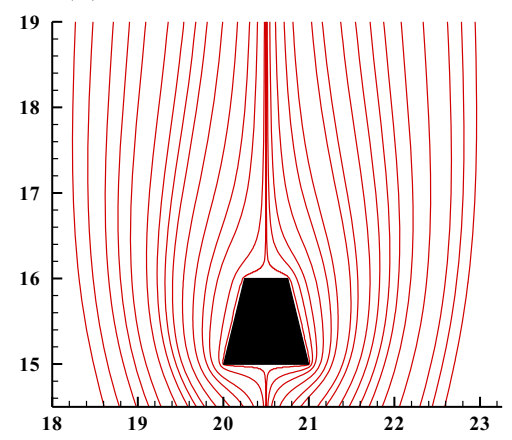

(b) $\mathbf{R i}=\mathbf{0}, \mathbf{R e}=\mathbf{2 0}$

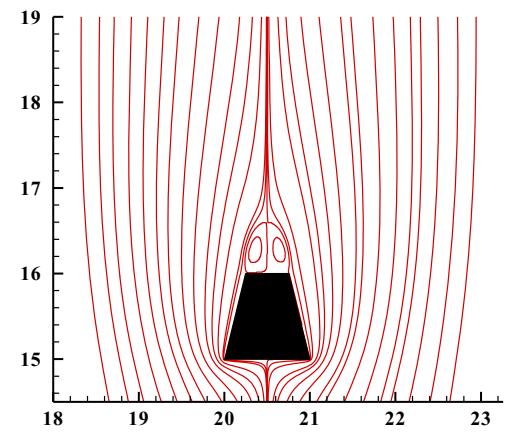

(c) $\mathrm{Ri}=\mathbf{0}, \mathbf{R e}=\mathbf{4 0}$

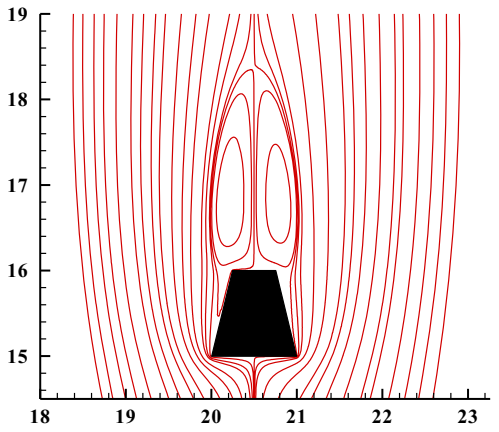

(d) $\mathrm{Ri}=0.5, \mathrm{Re}=5$

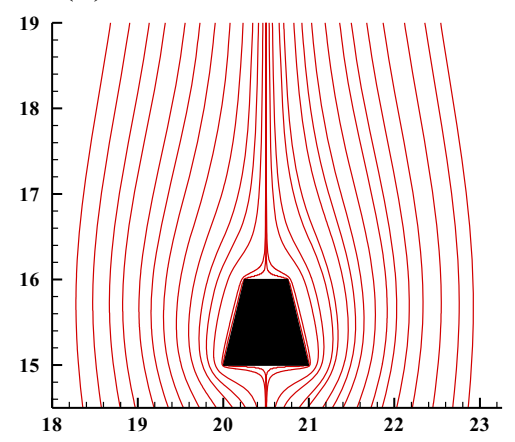

(e) $\mathrm{Ri}=0.5, \operatorname{Re}=20$

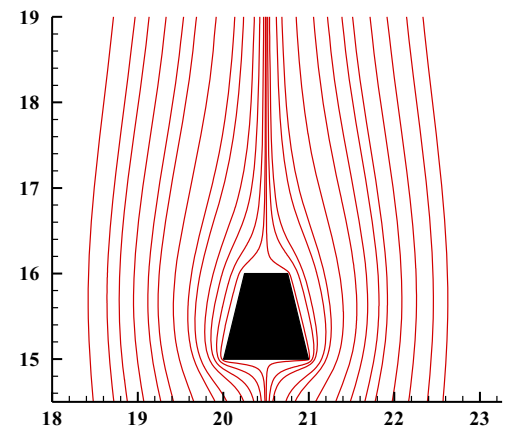

(f) $\mathrm{Ri}=0.5, \operatorname{Re}=40$

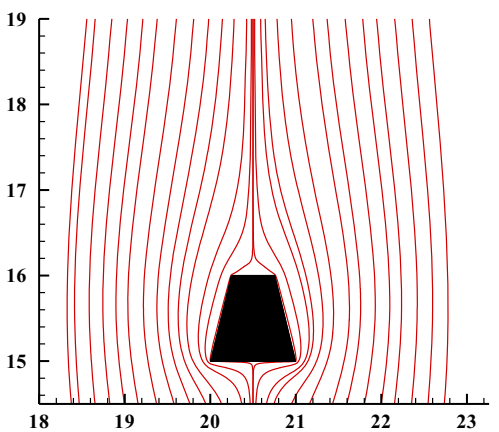

(g) $R \mathbf{i}=\mathbf{1}, \mathbf{R e}=5$

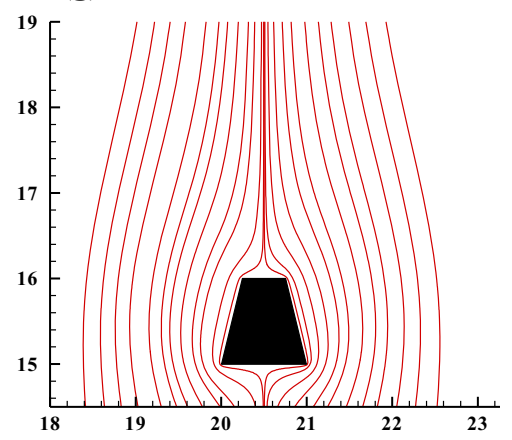

(h) $\mathrm{Ri}=\mathbf{1}, \mathbf{R e}=\mathbf{2 0}$

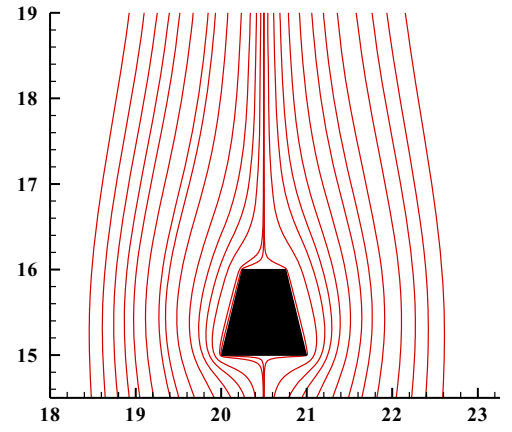

(i) $\mathbf{R i}=\mathbf{1}, \mathbf{R e}=\mathbf{4 0}$

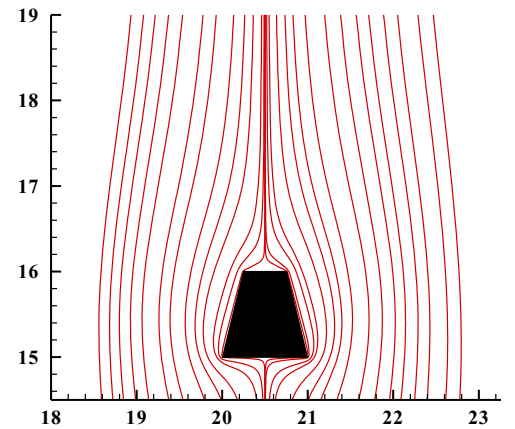

Figure 4. Streamlines around the converging trapezoidal cylinder at $\mathrm{Re}=5-40$ and $\mathrm{Ri}=0,0.5$ and 1 .

buoyancy mixed convection. In case of diverging trapezoidal cylinder, the variation in wake length for $\mathrm{Ri}=0$ is about $76 \%, 102 \%, 128 \%$ and $152 \%$ higher with those at $\mathrm{Ri}=0.5$ for $\mathrm{Re}=10,20,30$ and 40 , respectively. While comparing the variation of wake length for $\mathrm{Ri}=0.5$ with those at $\mathrm{Ri}=1$, the difference remains almost constant, in the range 22 to $25 \%$. In case of converging trapezoidal cylinder, the variation in $L r / B$ for $\mathrm{Ri}=0$ with respect to $\mathrm{Ri}=0.5$ is found to be the very large as can be visualised from figure 3. While comparing the values of $L r / B$ for $\mathrm{Ri}=0.5$ with respect to $\mathrm{Ri}=1$, the difference is in the range 70 to $80 \%$.

The following $L r / B-R i-R e$ correlation has been developed to calculate recirculation length for the diverging trapezoidal cylinder.

$$
L r / B=(0.0958-0.0675 R i) \operatorname{Re}^{0.83}\left(R^{2}=0.91\right)
$$

Equation (5) is valid in the range of $\mathrm{Ri}=0-1$ and $\mathrm{Re}$ up to 40. For converging trapezoidal cylinder, the wakes do not develop for low values of Re and due to the limited number of data points, wake length correlation is not developed.

Figures 4 and 5 depict streamlines in the vicinity of the converging and diverging trapezoidal cylinders at $\operatorname{Re}=5$, 20,40 and $\mathrm{Ri}=0,0.5,1$. The flow is steady for $\mathrm{Re}=5-40$ at $\mathrm{Ri}=0,0.5,1$ and the wake length has the significant dependence on Ri over the range of Re at fixed Pr. With the increase in $\mathrm{Re}$ the flow separation occurs near the rear end of the trapezoidal cylinders resulting in the formation of two vortices behind the object. Figures 4 and 5 also show that the wake behind the converging and diverging trapezoidal cylinders grows with the increase in Re at a particular value of $\mathrm{Ri}$ and $\mathrm{Pr}$, and which is consistent with available results $[9,20]$. The wake region decreases as Ri increases. This behaviour is more prominent in the 
(a) $\mathrm{Ri}=\mathbf{0}, \mathbf{R e}=\mathbf{5}$

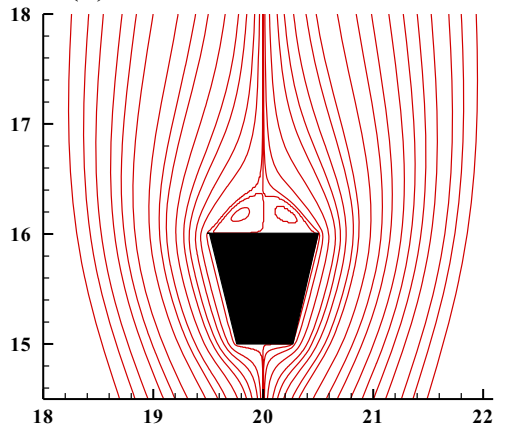

(b) $\mathbf{R i}=\mathbf{0}, \mathbf{R e}=\mathbf{2 0}$

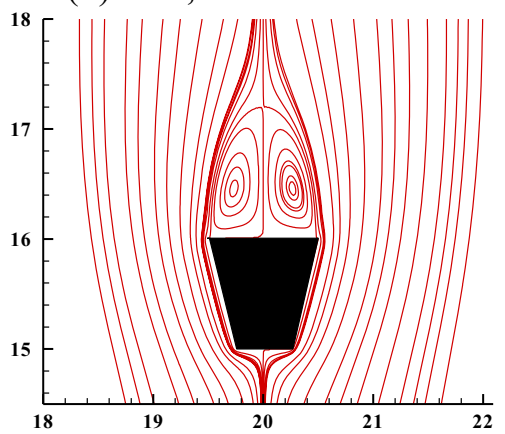

(c) $\mathbf{R i}=\mathbf{0}, \mathbf{R e}=\mathbf{4 0}$

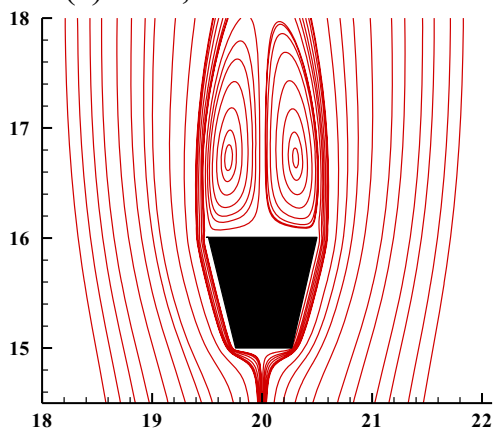

(d) $\mathrm{Ri}=0.5, \mathrm{Re}=5$

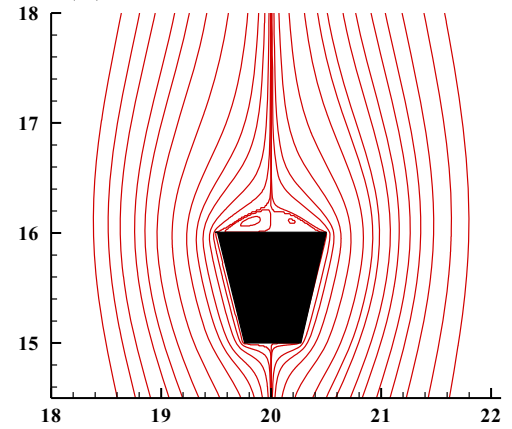

(e) $\mathrm{Ri}=0.5, \mathrm{Re}=\mathbf{2 0}$

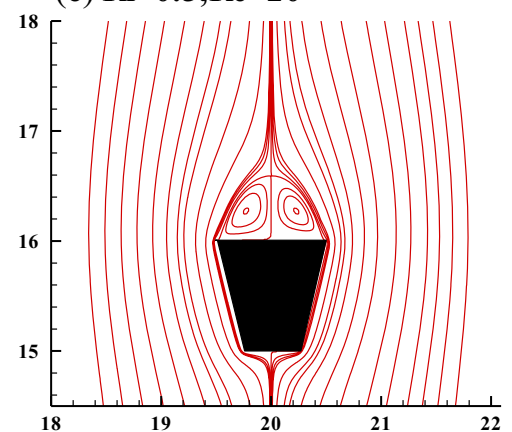

(f) $\mathbf{R i}=\mathbf{0 . 5}, \mathbf{R e}=\mathbf{4 0}$

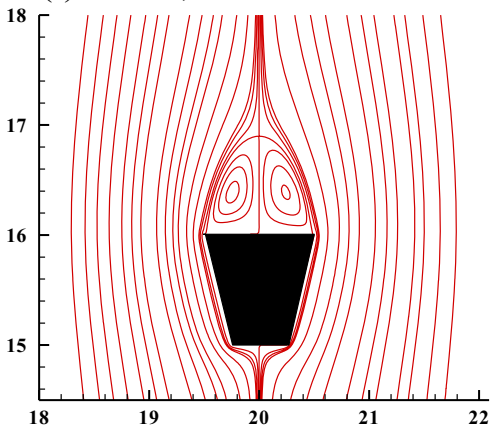

(g) $\mathbf{R i}=\mathbf{1}, \mathbf{R e}=\mathbf{5}$

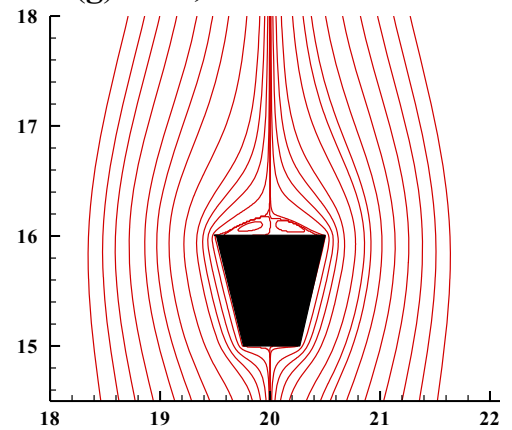

(h) $\mathbf{R i}=\mathbf{1}, \mathbf{R e}=\mathbf{2 0}$

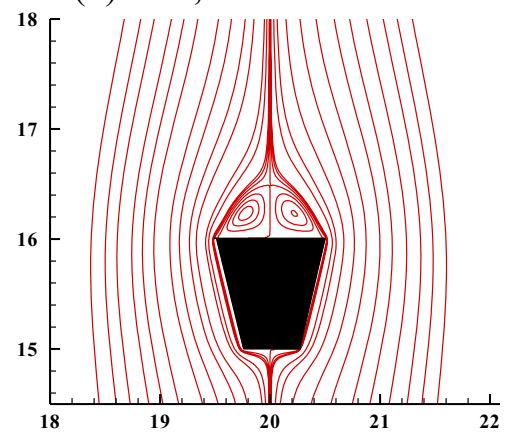

(i) $\mathbf{R i}=\mathbf{1}, \mathbf{R e}=\mathbf{4 0}$

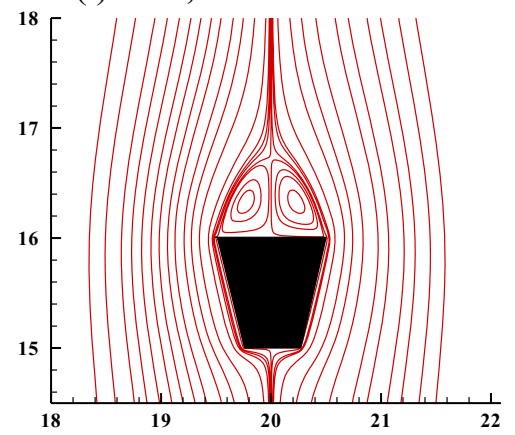

Figure 5. Streamlines around the diverging trapezoidal cylinder at $\mathrm{Re}=5-40$ and $\mathrm{Ri}=0,0.5$ and 1 .

diverging trapezoidal cylinder (because of large width on the rear side of the object), which results in the formation of larger size wake as compared to converging trapezoidal cylinder. This is in agreement with findings of [4]. Velocity in wake region increases as $\mathrm{Ri}$ increases which causes the shear layer weakening that decreases the width of the wake. The velocity adjacent to the surface of trapezoidal cylinders increases, which reduces the pressure in the vicinity of these cylinders. For $\mathrm{Ri}=0$ as the value of Re increasing the flow separation point moves from rear to front end of the trapezoidal cylinder. However, for $\mathrm{Ri}=0.5$ and 1 the flow separates at the rear end of the trapezoidal cylinder.

\subsection{Isotherm patterns}

Figures 6(a)-6(i) and 7(a)-7(i) show the effect of the control parameters ( $\mathrm{Re}$ and $\mathrm{Ri}$ ) on isotherms close to converging and diverging trapezoidal cylinders for the buoyancy aided air flow. The temperature contours are denser for smaller values of Re due to strong viscous effect. It is evident that as $\operatorname{Re}$ is low, heat is predominantly transferred through conduction. At higher Re values, the temperature contours spread with reduction of the lateral width of contours. The isotherms are concentrated more on those portions where the flow is attached. For a particular value of Ri, as Re increases, the density of isotherms in the upstream direction becomes prominent i.e., stemming an increase in heat transfer. The isotherms on the rear side are more congested for converging trapezoidal cylinder as compared with that of the diverging trapezoidal geometry, because of the difference in the widths on the rear side of the trapezoidal cylinders. The isotherms are more pointed in nature on the downstream of the converging cylinder as the shape of the rear edge of trapezoidal cylinder 
(a) $\mathrm{Ri}=0, \mathrm{Re}=5$

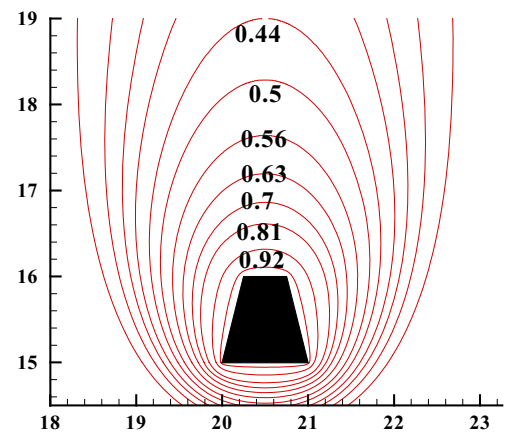

(b) $\mathrm{Ri}=\mathbf{0}, \mathbf{R e}=\mathbf{2 0}$

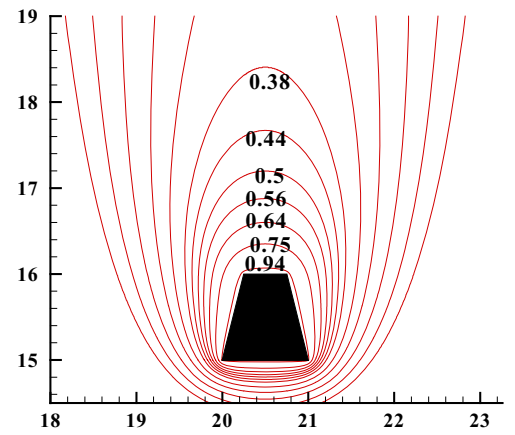

(c) $\mathrm{Ri}=\mathbf{0}, \mathbf{R e}=\mathbf{4 0}$

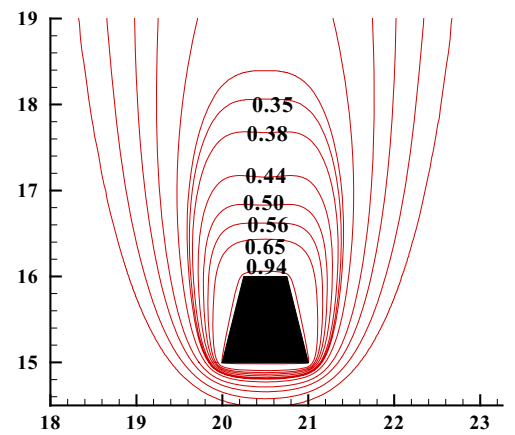

(d) $\mathrm{Ri}=0.5, \mathrm{Re}=5$

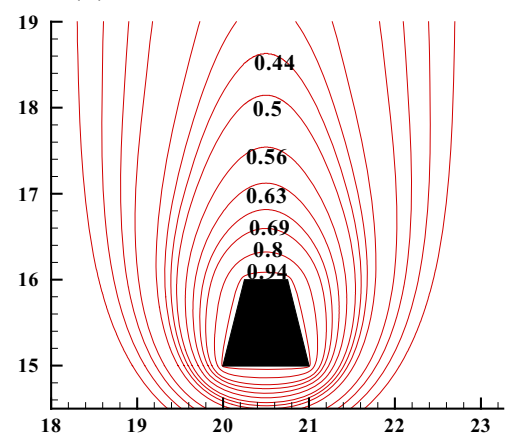

(e) $\mathrm{Ri}=0.5, \mathrm{Re}=\mathbf{2 0}$

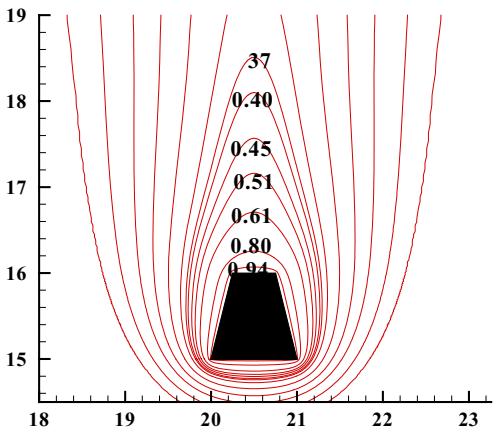

(f) $\mathrm{Ri}=0.5 \mathrm{Re}=40$

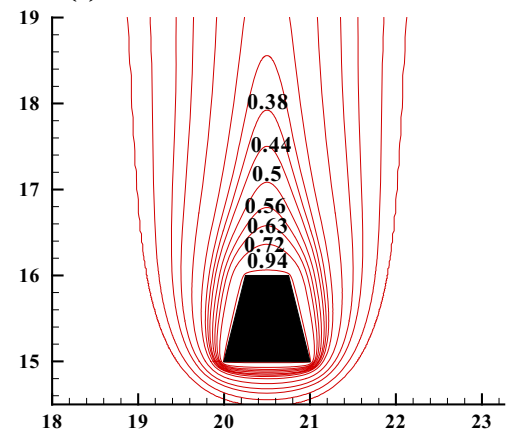

(g) $\mathrm{Ri}=1, \mathrm{Re}=5$

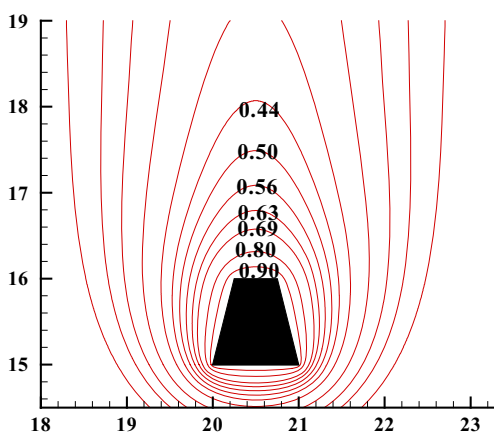

(h) $\mathrm{Ri}=\mathbf{1}, \mathbf{R e}=\mathbf{2 0}$

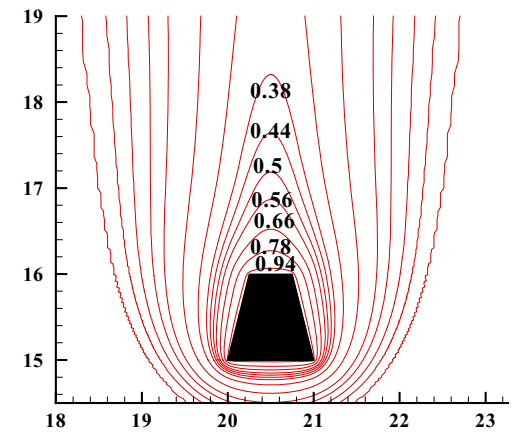

(i) $\mathrm{Ri}=\mathbf{1}, \mathbf{R e}=\mathbf{4 0}$

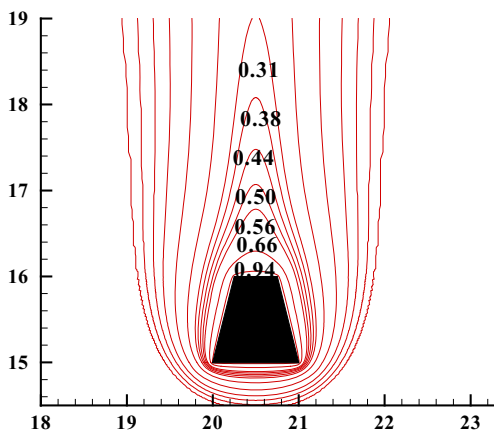

Figure 6. Isotherm patterns around the converging trapezoidal cylinder at $\operatorname{Re}=5-40$ and $\mathrm{Ri}=0,0.5$ and 1 .

substantially influences the isotherm patterns. The conspicuous pointed shape of isotherm patterns at the central region (on the rear side of the object) increases as a function of Re and Ri. The boundary layer growth starts from the front surface, indicating the highest Nusselt number. It then diminishes towards the right and left surfaces, and subsequently at the rear surface of the trapezoidal cylinder, which is in conformity with previous works $[4,9,20]$. This indicates that there is the maximum transfer of heat on the front face of the trapezoidal cylinders followed by the right and left faces of the trapezoidal cylinders and the least heat transfer is from the rear face of the trapezoidal cylinders.

\subsection{Drag coefficients}

The combination of frictional (viscous) drag coefficient and pressure (foam) drag coefficient constitutes the total drag coefficient $\left(C_{D}=C_{D p}+C_{D v}\right)$. Figures 8 and 9 provide individual (i.e., frictional and pressure drag coefficients) and overall drag coefficients and their comparison with the available literature $[9,10,17,18,20]$ under aiding buoyancy condition for both converging and diverging trapezoidal cylinders. $C_{D p}$ is comparatively higher than $C_{D v}$ for the range of $\mathrm{Ri}$ (figure 8). Further, it can be observed from figures 8 and 9 that individual and total drag coefficients are not linearly varying with $\mathrm{Re}$ and at different values of $\mathrm{Ri}$. Individual and overall drags show an inverse relationship with Re i.e., $C_{D v}, C_{D p}$ and $C_{D}$ decrease with increase in $\mathrm{Re}$ for fixed Ri. However, $\mathrm{C}_{\mathrm{D}}$ increases as Ri rises from 0 to 1. This is in agreement with the previous works $[4,9,10,20-22]$. On drawing the comparison, it is found (in figure 9(d)) that the square cylinder got the highest value of total drag coefficient followed by the diverging trapezoidal cylinder and the converging trapezoidal 
(a) $\mathbf{R i}=\mathbf{0}, \mathbf{R e}=\mathbf{5}$

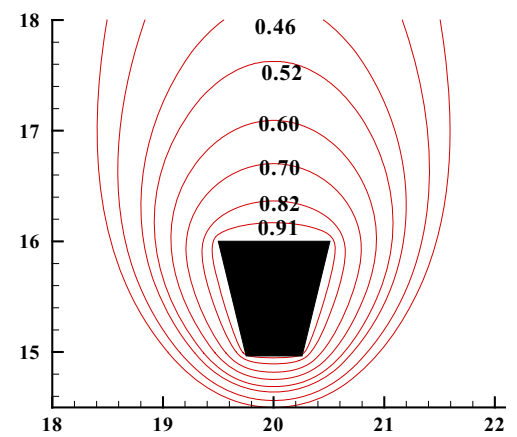

(b) $\mathrm{Ri}=\mathbf{0}, \mathbf{R e}=\mathbf{2 0}$

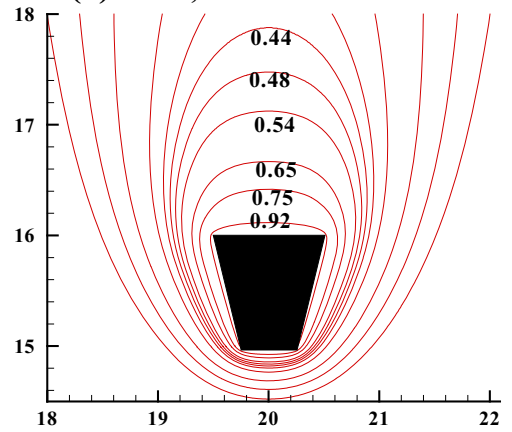

(c) $\mathrm{Ri}=\mathbf{0}, \mathbf{R e}=40$

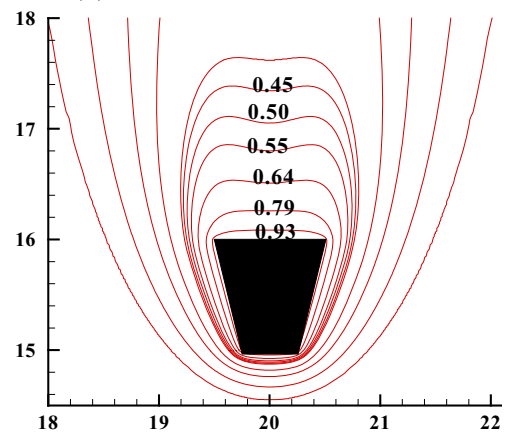

(d) $\mathrm{Ri}=0.5, \operatorname{Re}=5$

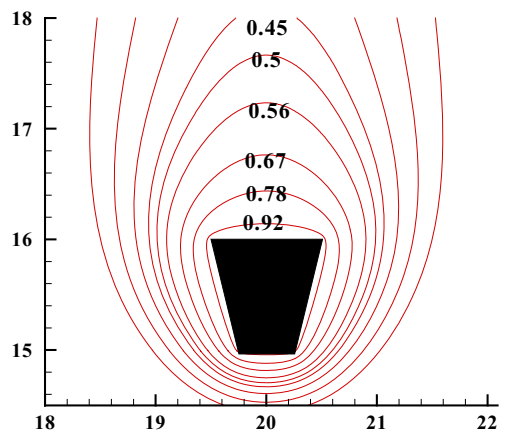

(e) $\mathrm{Ri}=0.5, \operatorname{Re}=20$

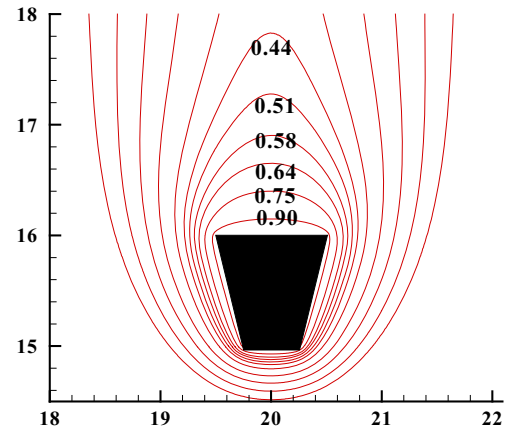

(f) $\mathrm{Ri}=0.5, \operatorname{Re}=40$

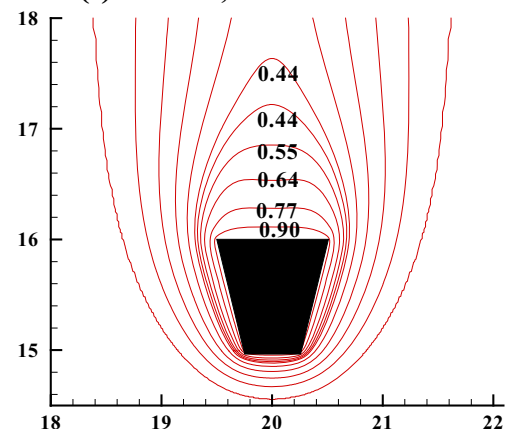

(g) $\mathrm{Ri}=1, \mathrm{Re}=5$

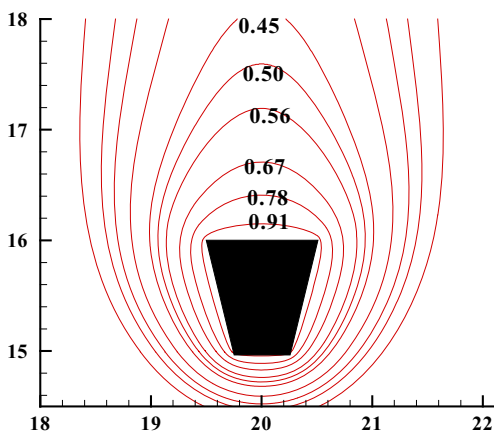

(h) $\mathrm{Ri}=\mathbf{1}, \mathbf{R e}=\mathbf{2 0}$

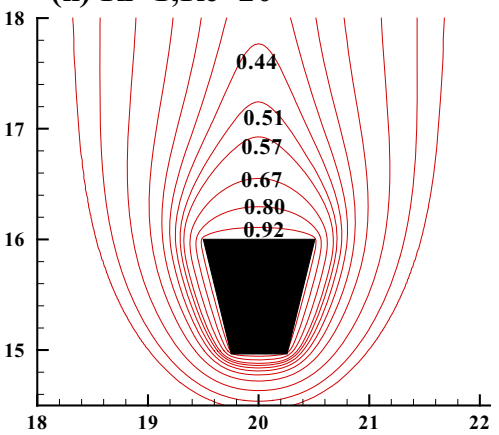

(i) $\mathrm{Ri}=1, \operatorname{Re}=40$

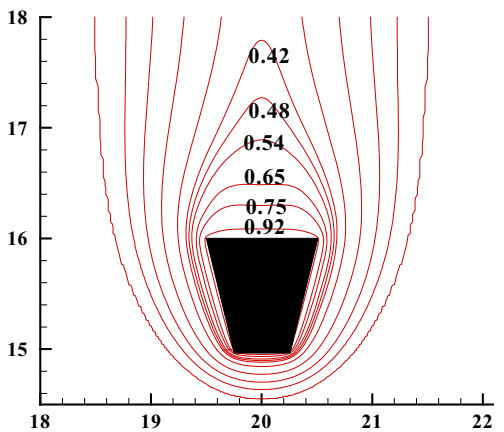

Figure 7. Isotherm patterns around the diverging trapezoidal cylinder at $\mathrm{Re}=5-40$ and $\mathrm{Ri}=0.5$ and 1 .

cylinder has the least. The total drag coefficient of the converging and diverging cylinders overlaps with the results of the same configuration i.e., converging and diverging cylinders $[17,18]$ for $\mathrm{Ri}=0$. The variation of $\mathrm{C}_{\mathrm{Dp}}$ with $\mathrm{Re}$ is almost similar to that of variation of overall drag coefficient. $C_{D v}$ values for diverging trapezoidal and circular cylinders are comparatively on the higher side than that of converging trapezoidal and square cylinders. The main reason for this difference is the variation in the shape of the trapezoidal cylinders as well as the change in the width of the front surface of the trapezoidal cylinders. Subsequently, as the Ri increases (as shown in figures 9(e)(9(f)) the drag coefficient of diverging cylinder starts to increase. With the increase in $\mathrm{Ri}$, velocity gradients increase, the streamlines converge adjacent to the back of the object causing the increase in viscous drag coefficient. Overall the $C_{D}$ increases with $\mathrm{Ri}$ and the drag for the square cylinder is the highest as compared with circular and trapezoidal cylinders under similar conditions. The present results have an excellent concurrence with the available results [9, 17, 18, 20, 21].

The buoyancy effects superimposed on the forced flow condition can strongly influence the flow patterns and therefore influence the convective heat transfer. If the flow is oriented normal to the direction of gravity under cross stream buoyancy, such cross buoyancy flow is highly complex because the velocity induced by the (thermal) buoyancy is normal to the flow direction and thus distort the flow field near the body. Consequently, this alters the values of drag coefficient and heat transfer irrespective of Re. 

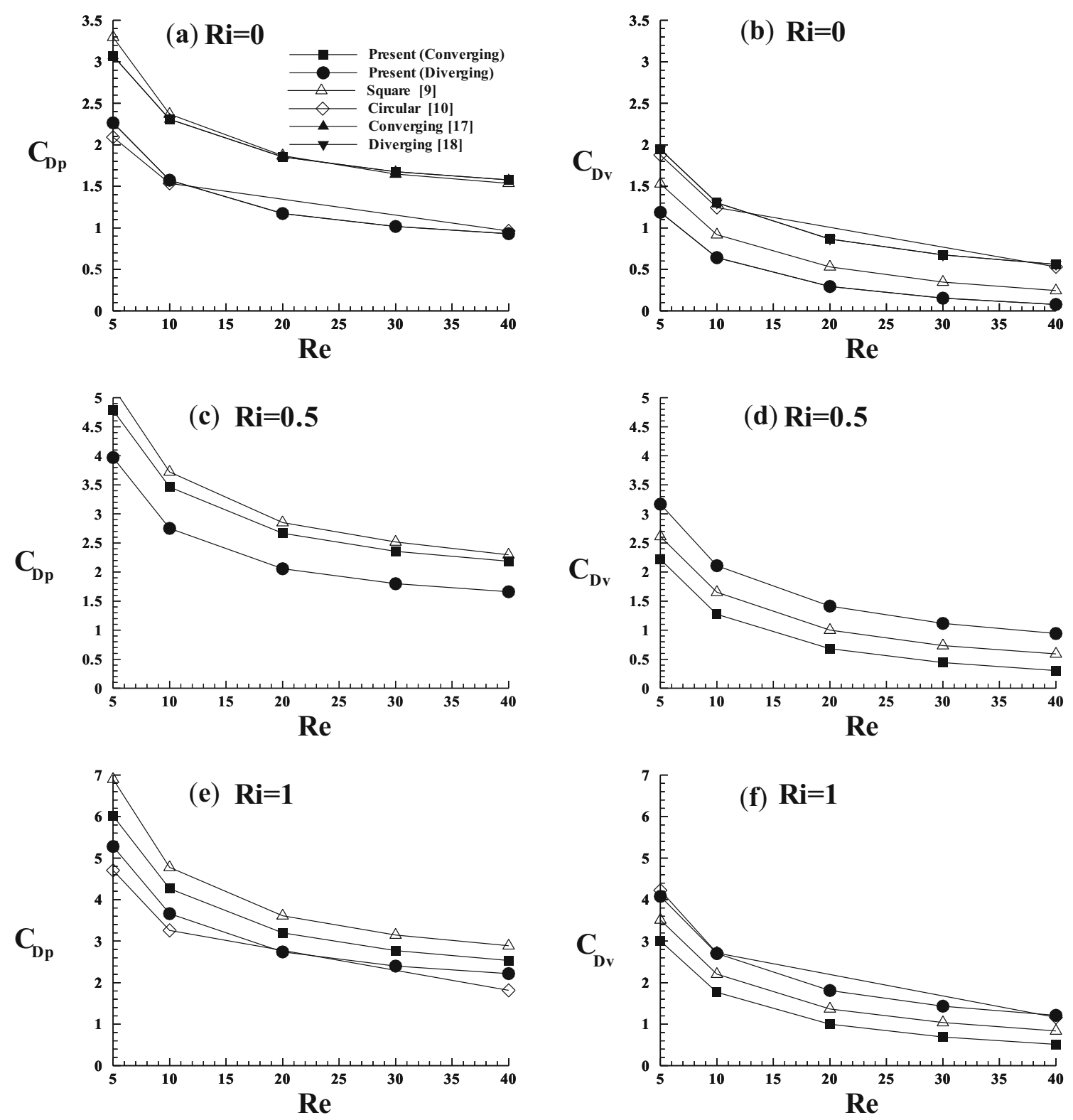

Figure 8. Comparison of pressure and viscous drag coefficients of trapezoidal, square and circular cylinders (at various Re).

It can be summed up that the previous studies [20,21] were carried out under cross flow condition. In these studies, it was observed that as $\mathrm{Ri}$ is increased there is a small change in the values of $C_{D p}, C_{D v}$ and $C_{D}$. Although, in the present case (aiding buoyancy), as $\mathrm{Ri}$ is increased the values of $C_{D p}, C_{D v}$ and $C_{D}$ show significant change.

\subsection{Local and average Nusselt numbers}

Figure 10 depicts the change of the local Nusselt number $\mathrm{Nu}_{\mathrm{L}}$ on the faces of the 2-D converging and diverging trapezoidal cylinders for various $\mathrm{Re}$ and $\mathrm{Ri}$ values covered with air as working fluid. Under identical conditions, the local Nusselt number for $\mathrm{Ri}=0$ is lower than the mixed convection cases with $\mathrm{Ri}=0.5,1$. This is due to change in thermal gradients with Ri. There is the marginal change in $\mathrm{Nu}_{\mathrm{L}}$ with respect to the increase in the values of $\mathrm{Ri}$ i.e., from 0.5 to 1 . It is also observed (from figures 10(a)-10(f)) that because of maximum congestion of isotherms on the front surface of the trapezoidal cylinders, the $\mathrm{Nu}_{\mathrm{L}}$ is the highest near the front face, the lowest near the rear/top face and intermediate on the left and right faces of the converging and diverging trapezoidal cylinders. There is no difference in $\mathrm{Nu}_{\mathrm{L}}$ on the right and left faces because of symmetrical shape.

The average Nusselt number $\overline{N u}$ of the converging and diverging trapezoidal cylinders with $\mathrm{Re}$ and $\mathrm{Ri}$ at $\mathrm{Pr}=0.71$ 

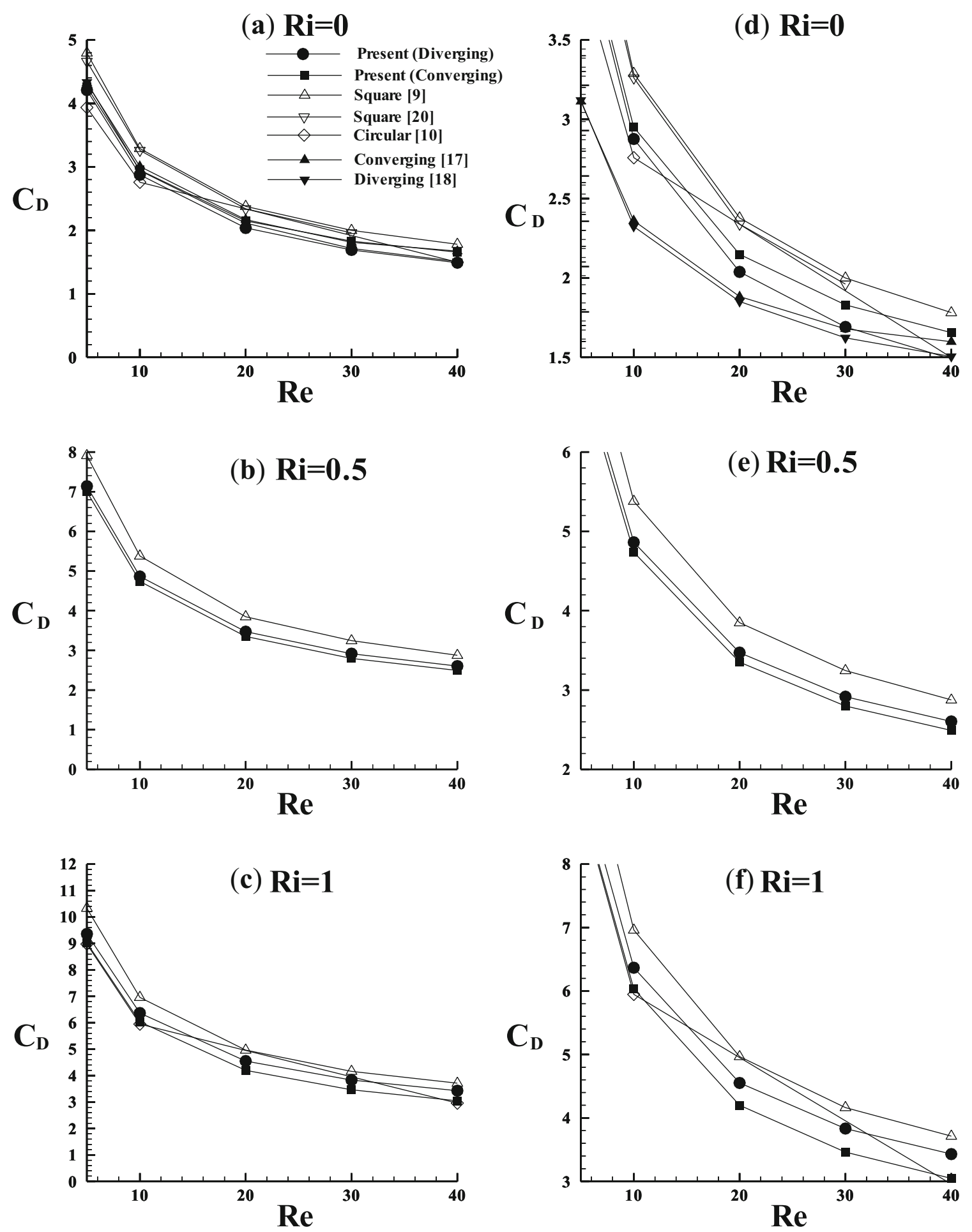

Figure 9. Comparison of total drag coefficients of trapezoidal, square and circular cylinders (at various Re).

is presented in figures $11(\mathrm{a})-11(\mathrm{c})$. The present results are compared with analogous geometry i.e., converging [17] and diverging [18] cylinders. It is pertinent to mention that these results $[17,18]$ are available only for $\mathrm{Ri}=0$ and show excellent concurrence with the present investigation. Also, the present results are compared with the data available for circular and square cylinders in literature $[9,10,20]$ in the steady regime (under the similar flow conditions). Further it is necessary to mention that the results obtained in $[9,20]$ are for $\operatorname{Pr}=0.7$ and in [10] for $\operatorname{Pr}=1$, whereas the present results are expressed for $\operatorname{Pr}=0.71 . \overline{\mathrm{Nu}}$ increases with the increase in Re and also by 
(a) $\operatorname{Re}=5$

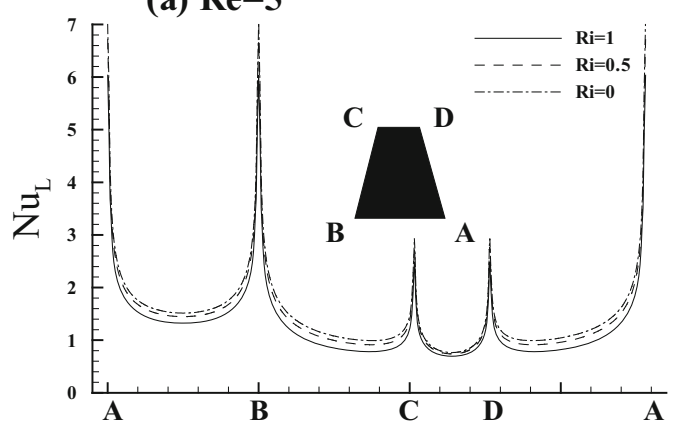

(b) $\operatorname{Re}=\mathbf{2 0}$

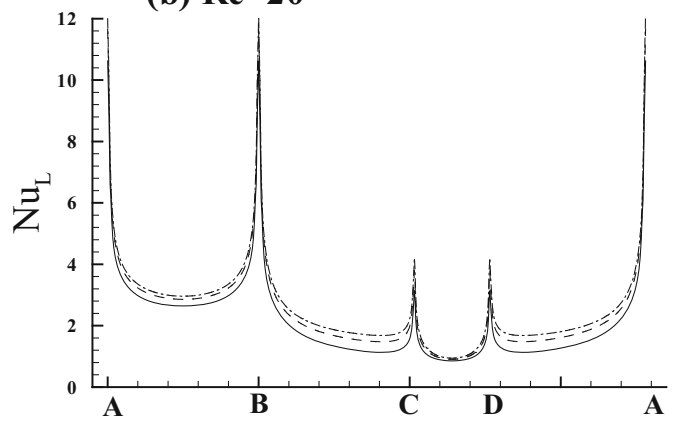

(c) $\mathbf{R e}=40$

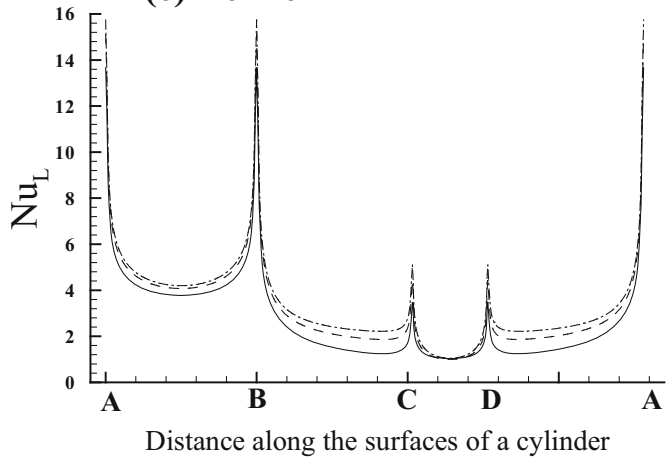

(d) $\mathrm{Re}=5$

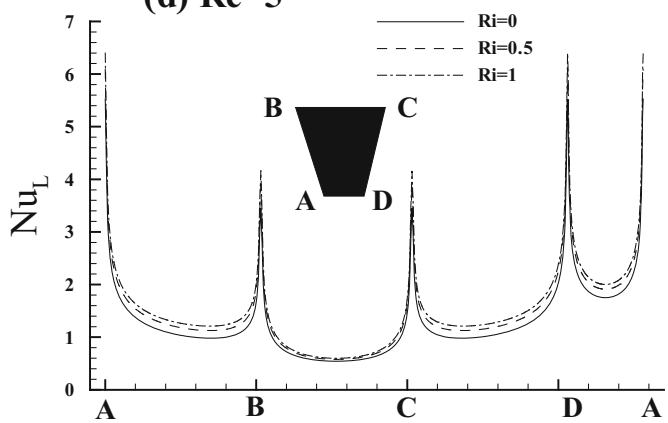

(e) $\operatorname{Re}=\mathbf{2 0}$

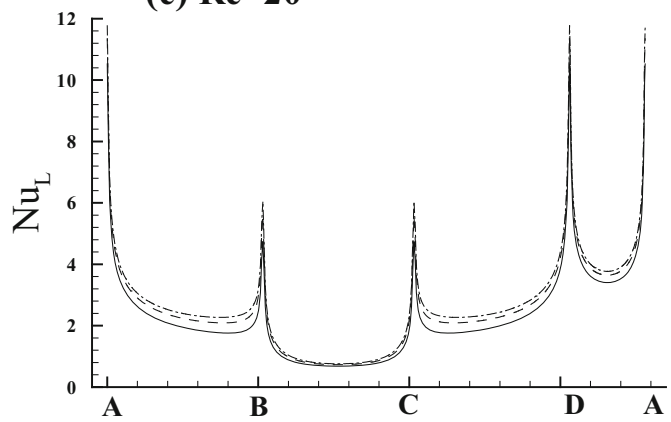

(f) $\mathbf{R e}=\mathbf{4 0}$

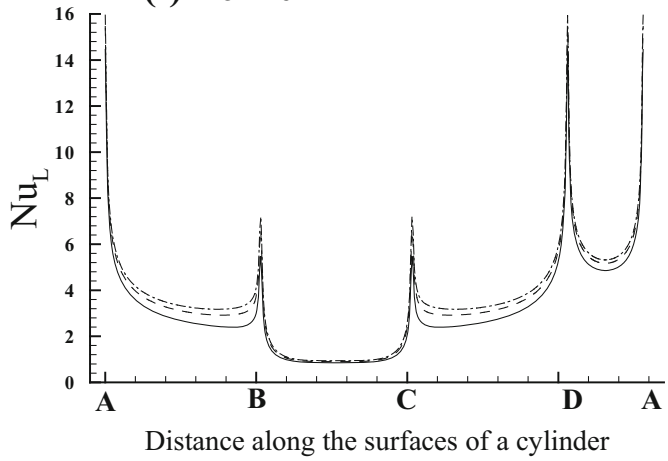

Figure 10. Variation of local Nusselt number around the faces of diverging and converging trapezoidal cylinders at different Re and Ri.

increasing Ri. Under the influence of buoyancy, $\overline{N u}$ is always higher than at $\mathrm{Ri}=0$ (as is observed for the converging and diverging trapezoidal cylinders, table 6). It has also been observed that at higher $\mathrm{Re}$, the effect of $\mathrm{Ri}$ is more prominent. At a particular Ri there is increase in the enhancement of heat transfer with change in Re. The comparison of present results with [9] for square and diverging trapezoidal cylinders shows that the enhancement in heat transfer is around $14.6 \%$ at $\mathrm{Re}=5$ and $23 \%$ at $\mathrm{Re}=40$ for the fixed $\mathrm{Ri}=0$, as given in table 6 . The similar behaviour is shown by square [20] and circular [10] cylinders. However, it is necessary to mention that the value of $\overline{N u}$ for the circular cylinder is the highest as compared to the present converging and diverging trapezoidal cylinders and also with that of square cylinder considered in $[9,20]$ under the same set of conditions. Furthermore, under aiding buoyancy condition, the value of
$\overline{N u}$ for the diverging trapezoidal cylinder is higher as compared to that of the converging trapezoidal cylinder. Height of the front surface of the trapezoidal cylinders affect the local heat transfer coefficient significantly. The converging cylinder has got high value of $\mathrm{Nu}_{\mathrm{L}}$ at low Re, as shown in figures 10(a) and 10(d). As Re increases, the local heat transfer remains almost same in both configurations, as shown in figures 10(c) to 10(f). However, the reverse behaviour is observed while determining the value of average Nusselt number. If the flow remains attached with maximum number of faces, higher will be the value of $\overline{\mathrm{Nu}}$. In case of the diverging cylinder, the front face is small when compared to the converging cylinder so the tendency of the flow is to remain attached to the face, resulting in the larger value of $\overline{N u}$. Finally, $\overline{N u}$ is correlated with Re and Ri for the converging and diverging trapezoidal cylinders in the range of $5<\mathrm{Re} \leq 40$ and $\mathrm{Ri}=0-1$. 

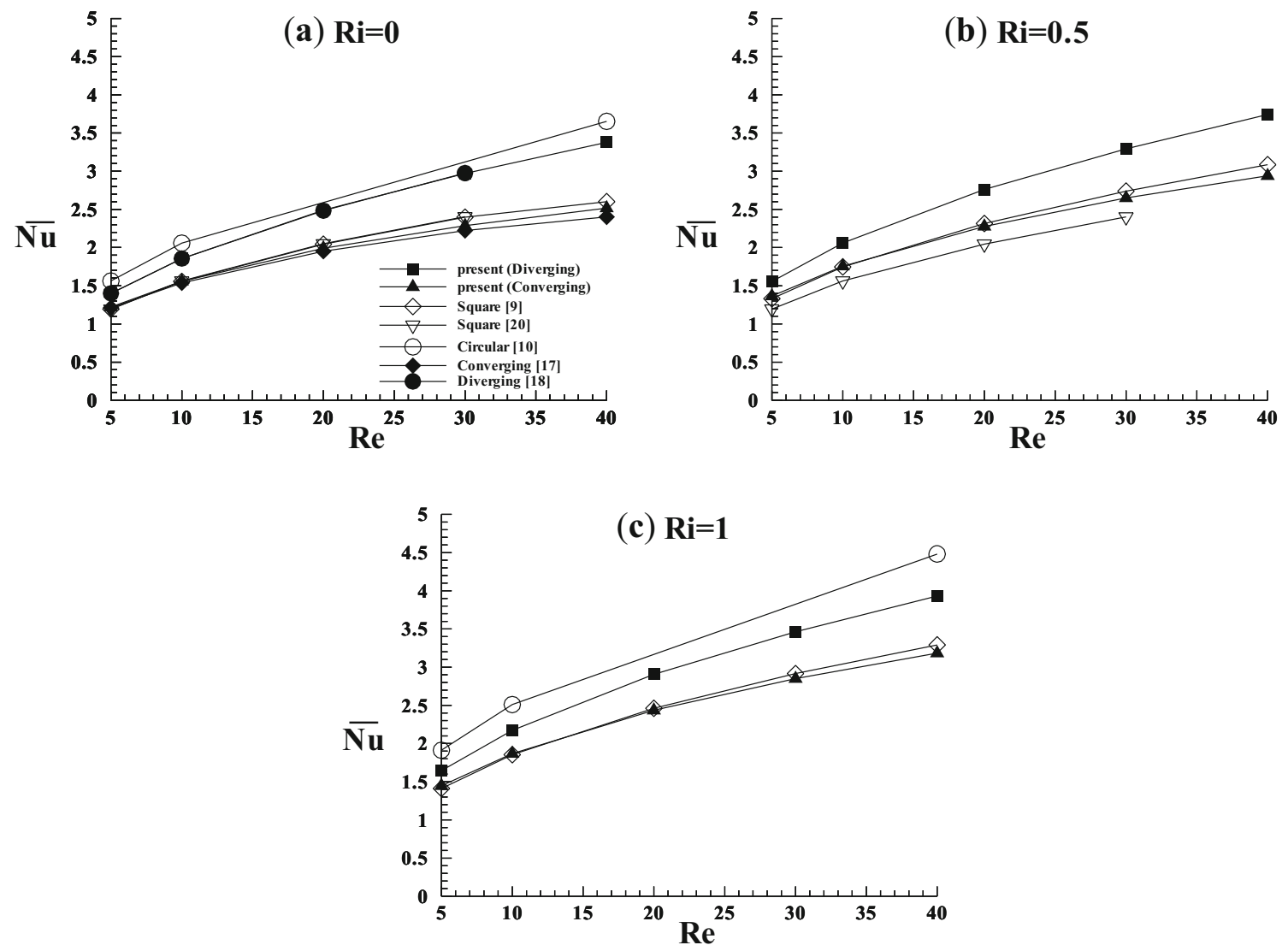

Figure 11. Variation of average Nusselt number of various cylinders at different values of Re and Ri and comparison with literature $[9,10,17,18,20]$.

Table 6. Heat transfer enhancement of both converging and diverging cylinders as compared to square and circular cylinders [9, 10, 20] for $\mathrm{Ri}=0-1$ and $\mathrm{Re}=5-40$.

\begin{tabular}{|c|c|c|c|c|c|c|c|c|c|c|c|c|}
\hline \multirow[b]{2}{*}{$\operatorname{Re}$} & \multirow[b]{2}{*}{$\begin{array}{c}\text { Present } \\
\text { (Conv.* } \\
\text { cylinder) }\end{array}$} & \multirow[b]{2}{*}{$\begin{array}{l}\text { Present } \\
\text { (Divr. } \\
\text { cylinder) }\end{array}$} & \multirow[b]{2}{*}{$\begin{array}{c}\text { Square } \\
{[20]}\end{array}$} & \multirow[b]{2}{*}{$\begin{array}{c}\text { Square } \\
{[9]}\end{array}$} & \multirow[b]{2}{*}{$\begin{array}{c}\text { Circular } \\
{[10]}\end{array}$} & \multicolumn{3}{|c|}{ Conv.* cylinder w.r.t. } & \multirow{2}{*}{$\begin{array}{c}\text { Divr. }^{\$} \\
\text { cylinder } \\
\text { w.r.t. conv.* } \\
\text { cylinder }\end{array}$} & \multicolumn{3}{|c|}{ Divr. $^{\$}$ cylinder w.r.t. } \\
\hline & & & & & & $\begin{array}{c}\text { Square } \\
{[20]}\end{array}$ & $\begin{array}{c}\text { Square } \\
\text { [9] }\end{array}$ & $\begin{array}{c}\text { Circular } \\
\text { [10] }\end{array}$ & & $\begin{array}{c}\text { Square } \\
{[20]}\end{array}$ & $\begin{array}{c}\text { Square } \\
{[9]}\end{array}$ & $\begin{array}{c}\text { Circular } \\
\text { [10] }\end{array}$ \\
\hline 5 & 1.2212 & 1.3992 & 1.1937 & 1.1950 & $\begin{array}{c}\mathrm{Ri}=0 \\
1.5619\end{array}$ & 2.30 & 2.18 & -21.82 & 12.73 & 14.69 & 14.59 & -11.63 \\
\hline 10 & 1.5581 & 1.8553 & 1.5624 & 1.5573 & 2.0580 & -0.27 & 0.05 & -24.29 & 16.02 & 15.79 & 16.06 & -10.93 \\
\hline 20 & 1.9909 & 2.4895 & 2.0495 & 2.0436 & - & -2.8 & -2.58 & - & 20.03 & 17.67 & 17.91 & - \\
\hline 30 & 2.2870 & 2.9713 & 2.4039 & 2.3978 & - & -4.87 & -4.62 & - & 23.03 & 19.09 & 19.30 & - \\
\hline 40 & 2.5166 & 3.3776 & 2.6969 & 2.6012 & $\begin{array}{c}3.6526 \\
\mathrm{Ri}=0.5\end{array}$ & -6.68 & -3.52 & -31.10 & 25.49 & 20.15 & 22.99 & -8.14 \\
\hline 5 & 1.3649 & 1.5559 & 1.1952 & 1.3309 & - & 14.19 & 2.55 & - & 2.45 & 14.58 & 4.88 & - \\
\hline 10 & 1.7560 & 2.0601 & 1.5625 & 1.7473 & - & 12.39 & 0.49 & - & 5.35 & 15.78 & 5.82 & - \\
\hline 20 & 2.2770 & 2.7608 & 2.0458 & 2.3145 & - & 11.30 & -1.62 & - & 8.54 & 17.82 & 7.03 & - \\
\hline 30 & 2.6499 & 3.2934 & 2.4017 & 2.7393 & - & 10.34 & -3.27 & - & 10.82 & 19.17 & 7.81 & - \\
\hline 40 & 2.9394 & 3.7428 & - & 3.0855 & $\begin{array}{c}- \\
\mathrm{Ri}\end{array}$ & - & -4.74 & - & 12.97 & - & 8.65 & - \\
\hline 5 & 1.4470 & 1.6445 & - & 1.4114 & 1.9115 & - & 2.53 & -24.30 & 12.01 & - & 14.18 & -16.24 \\
\hline 10 & 1.8675 & 2.1732 & - & 1.8538 & 2.5099 & - & 0.73 & -25.60 & 14.07 & - & 14.7 & -15.49 \\
\hline 20 & 2.4352 & 2.9065 & - & 2.4615 & - & - & -1.07 & - & 16.21 & - & 15.31 & - \\
\hline 30 & 2.8494 & 3.4628 & - & 2.9154 & - & - & -2.26 & - & 17.71 & - & 15.81 & - \\
\hline 40 & 3.1829 & 3.9316 & - & 3.2917 & 4.4812 & - & 3.31 & -28.97 & 19.04 & - & 16.28 & -13.98 \\
\hline
\end{tabular}

*Conv.: Converging

$\$$ Divr.: Diverging 
Converging trapezoidal cylinder:

$$
\overline{N u}=(0.6621+0.1553 R i) \operatorname{Re}^{0.3689}\left(R^{2}=0.99\right)
$$

Diverging trapezoidal cylinder:

$$
\overline{N u}=(0.7069+0.1168 R i) \operatorname{Re}^{0.4250}\left(R^{2}=0.99\right)
$$

\section{Concluding remarks}

Comparison of flow and thermal characteristics of converging and diverging trapezoidal infinite cylinders has been addressed, under aiding buoyancy condition $(\mathrm{Ri}=0$ 1) at $\operatorname{Re}=5-40$ and $\operatorname{Pr}=0.71$ in a vertical domain. With the introduction of $\mathrm{Ri}$, the wake length starts to decrease and is found to be higher for diverging trapezoidal cylinder as compared with that of converging trapezoidal cylinder. The overall drag coefficient increases with increase in $\mathrm{Ri}$ and the drag for the square cylinder is the highest as compared to circular and trapezoidal cylinders under similar flow conditions. The congestion of isotherms adjacent to front face of converging cylinder is comparatively higher than in case of diverging cylinder. There is insignificant change in $\mathrm{Nu}_{\mathrm{L}}$ with the change in Ri. The values of $\overline{\mathrm{Nu}}$ for the diverging trapezoidal cylinder are higher as compared with that of the converging trapezoidal cylinder. The enhancement in heat transfer increases with the increase in $\mathrm{Re}$ at a particular Ri. If a comparison is drawn with square cylinder [9] and diverging trapezoidal cylinder, the enhancement in heat transfer is around $14.6 \%$ (at $\mathrm{Re}=5$ ) and $23 \%$ (at $\operatorname{Re}=40$ ) for the fixed Richardson number $(\mathrm{Ri}=0)$. The values of $\overline{\mathrm{Nu}}$ for the circular cylinder are the highest as compared to the converging, diverging trapezoidal and square cylinders. Finally, $\overline{\mathrm{Nu}}$-Re-Ri correlations are established for the conditions covered in this work.

\section{Acknowledgement}

The authors would like to thank the editors and the reviewers for their valuable and helpful suggestions that have enriched the quality of the article.

\section{List of symbols}

$B \quad$ Front width of trapezium, $\mathrm{m}$

$C_{p} \quad$ Specific heat of fluid, $\mathrm{J} \mathrm{kg}^{-1} \mathrm{~K}^{-1}$

$\mathrm{CV}$ Control volume

$C_{D} \quad$ Overall drag coefficient $\left(=\frac{2 F_{D}}{\rho \nu_{\infty}^{2} B}=C_{D p}+C_{D v}\right)$

$C_{D p} \quad$ Pressure drag coefficient $\left(=\frac{2 F_{D p}}{\rho v_{\infty}^{2} B}\right)$

$C_{D v} \quad$ Viscous drag coefficient $\left(=\frac{2 F_{D v}}{\rho v_{\infty}^{2} B}\right)$

$F_{D p} \quad$ Pressure drag force on the object, $\mathrm{N} \mathrm{m}^{-1}$

$F_{D v} \quad$ Viscous drag force on the object, $\mathrm{N} \mathrm{m}^{-1}$
$F_{D} \quad$ Drag force on the object, $\mathrm{N} \mathrm{m}^{-1}$

$g \quad$ Acceleration because of gravity, $\mathrm{m} \mathrm{s}^{-2}$

Gr Grashof number $\left(=\frac{g \beta\left(T_{w}-T_{\infty}\right) B^{3}}{v^{2}}\right)$

$h \quad$ Local heat transfer coefficient, $\mathrm{W} \mathrm{m}^{-2} \mathrm{~K}^{-1}$

$\bar{h} \quad$ average heat transfer coefficient, $\mathrm{W} \mathrm{m}^{-2} \mathrm{~K}^{-1}$

$H_{u} \quad$ Upstream distance, $\mathrm{m}$

$H_{d} \quad$ Downstream distance, $\mathrm{m}$

$H_{T} \quad$ Total height, $\mathrm{m}$

$k \quad$ Coefficient of thermal conductivity, $\mathrm{W} \mathrm{m}^{-1} \mathrm{~K}^{-1}$

Lr Wake length, $\mathrm{m}$

$L_{1} \quad$ Domain length, $\mathrm{m}$

$N u_{L} \quad$ Nusselt number (local) $(=h B / k)$

$\overline{N u} \quad$ Average Nusselt number $(=\bar{h} B / k)$

$p$ Pressure, $\mathrm{N} \mathrm{m}^{-2}$

$P \quad$ Pressure $\left(=\frac{p}{\rho v_{\infty}^{2}}\right)$

Pr Prandtl number $\left(=\frac{\mu C_{p}}{k}\right)$

Re Reynolds number $\left(=\frac{\rho v_{\infty} B}{\mu}\right)$

Ri Richardson number $\left(=\frac{G r}{\operatorname{Re}^{2}}\right)$

$t$ Time, $\mathrm{s}$

$T$ Temperature, $\mathrm{K}$

$T_{w} \quad$ Constant wall temperature of solid surface, $\mathrm{K}$

$T_{\infty} \quad$ Stream temperature, $\mathrm{K}$

$u \quad$ Cross stream velocity, $\mathrm{m} \mathrm{s}^{-1}$

$U \quad$ Cross-stream velocity $\left(=u / v_{\infty}\right)$ (non-dimensional)

$v \quad$ Stream-wise velocity, $\mathrm{m} \mathrm{s}^{-1}$

$v_{\infty} \quad$ Inlet free-stream velocity, $\mathrm{m} \mathrm{s}^{-1}$

$V \quad$ Stream-wise velocity $\left(=v / v_{\infty}\right)$ (non-dimensional)

$x \quad$ Cross stream coordinate, $\mathrm{m}$

$X \quad$ Cross stream coordinate $(=x / B)$ (non-dimensional)

$y \quad$ Stream-wise coordinate, $\mathrm{m}$

$Y \quad$ Stream-wise coordinate $(=y / B)$ (non-dimensional)

$\beta \quad$ Coefficient of volumetric thermal expansion, $\mathrm{K}^{-1}$

$\delta \quad$ Size of the CV clustered around the object

$\theta \quad$ Dimensionless temperature $\left(=\frac{T-T_{\infty}}{T_{w}-T_{\infty}}\right)$

$\mu \quad$ Dynamic viscosity of the fluid, $\mathrm{kg} \mathrm{m}^{-1} \mathrm{~s}^{-1}$

$\rho \quad$ Density of the fluid, $\mathrm{kg} \mathrm{m}^{-3}$

$\tau \quad$ Time $\left(=t /\left(B / v_{\infty}\right)\right)$ (non-dimensional)

\section{References}

[1] Badr H M 1984 Laminar combined convection from a horizontal cylinder - parallel and counter flow regimes. Int. J. Heat Mass Transf. 27: 15-27

[2] Ho C J, Wu M S and Jou J B 1990 Analysis of buoyancyaided convection heat transfer from a horizontal cylinder in a vertical duct at low Reynolds number. Wärme und Stoffübertragung 25: 337-343

[3] Badr H M 1983 A theoretical study of laminar mixed convection from a horizontal cylinder in a cross stream. Int. J. Heat Mass Transf. 26: 639-653 
[4] Sharma A and Eswaran V 2004 Effect of aiding and opposing buoyancy on the heat and fluid flow across a square cylinder at $\mathrm{Re}=100$. Numer. Heat Transf. A 45: 601-624

[5] Singh S, Biswas G and Mukhopadhyay A 1998 Effect of thermal buoyancy on the flow through a vertical channel with a built-in circular cylinder. Numer. Heat Transf. A 34: 769-789

[6] Singh S K, Panigrahi P K and Muralidhar K 2007 Effect of buoyancy on the wakes of circular and square cylinders: a Schlieren-interferrometric study. Exp. Fluids 43: 101-123

[7] Maudou L, Choueiri G H and Tavoularis S 2013 An experimental study of mixed convection in vertical, open-ended, concentric and eccentric annular channels. J. Heat Transf. 135: 072502-072509

[8] Venugopal A, Agrawal A and Prabhu S V 2010 Influence of blockage and upstream disturbances on the performance of a vortex flow meter with a trapezoidal bluff body. Measurement 43: 603-616

[9] Sharma N, Dhiman A K and Kumar S 2012 Mixed convection flow and heat transfer across a square cylinder under the influence of aiding buoyancy at low Reynolds numbers. Int. J. Heat Mass Transf. 55: 2601-2614

[10] Srinivas A T, Bharti R P and Chhabra R P 2009 Mixed convection heat transfer from a cylinder in power-law fluids: effect of aiding buoyancy. Ind. Eng. Chem. Res. 48: 9735-9754

[11] Lee T S 1998 Early stages of an impulsively started unsteady laminar flow past tapered trapezoidal cylinders. Int. J. Numer. Meth. Fluids 26: 1181-1203

[12] Sun Z 2011 Design and performance of the converging-diverging vortex flow meter. Metrol. Measur. Syst. 18: $129-136$
[13] Chung Y J and Kang S H 2000 Laminar vortex shedding from a trapezoidal cylinder with different height ratios. Phys. Fluids 12: 1251-1254

[14] Verma V K and Dhiman A 2017 A comparative study on cross-buoyancy mixed convection around expanded and tapered trapezoidal bluff bodies. Proc. Inst. Mech. Engineers, Part E: J. Process Mech. Eng. 231: 513-532

[15] Parveez M, Dhiman A K and Rasool T 2015 Transition to periodic unsteady and effects of Prandtl and Richardson numbers on the flow across a confined heated trapezoidal prism. J. Braz. Soc. Mech. Sci. Eng. 37: 1291-1307

[16] Kahawita R and Wang P 2002 Numerical simulation of the wake flow behind trapezoidal bluff bodies. Comp. Fluids 31: 99-112

[17] Dhiman A K and Hasan M 2013 Flow and heat transfer over a trapezoidal cylinder: steady and unsteady regimes. Asia Pacific J. Chem. Eng. 8: 433-446

[18] Dhiman A K and Ghosh R 2013 Computer simulation of momentum and heat transfer across an expanded trapezoidal bluff body. Int. J. Heat Mass Transf. 59: 338-352

[19] ANSYS User Manual, Ansys, Inc., Canonsburg, PA, 2009

[20] Dhiman A K, Anjaiah N, Chhabra R P and Eswaran V 2007 Mixed convection from a heated square cylinder to Newtonian and power-law fluids. J. Fluids Eng. 129: 506-513

[21] Anjaiah N, Dhiman A K and Chhabra R P 2006 Mixed convection heat transfer from a square cylinder to power-law fluids in cross flow. In: ASME 2nd Joint U.S.-European Fluids Eng. Summer Meeting, Miami, FL

[22] Dhiman A K, Sharma N and Kumar S 2012 Wall effects on the cross-buoyancy around a square cylinder in the steady regime. Braz. J. Chem. Eng. 29: 253-264 